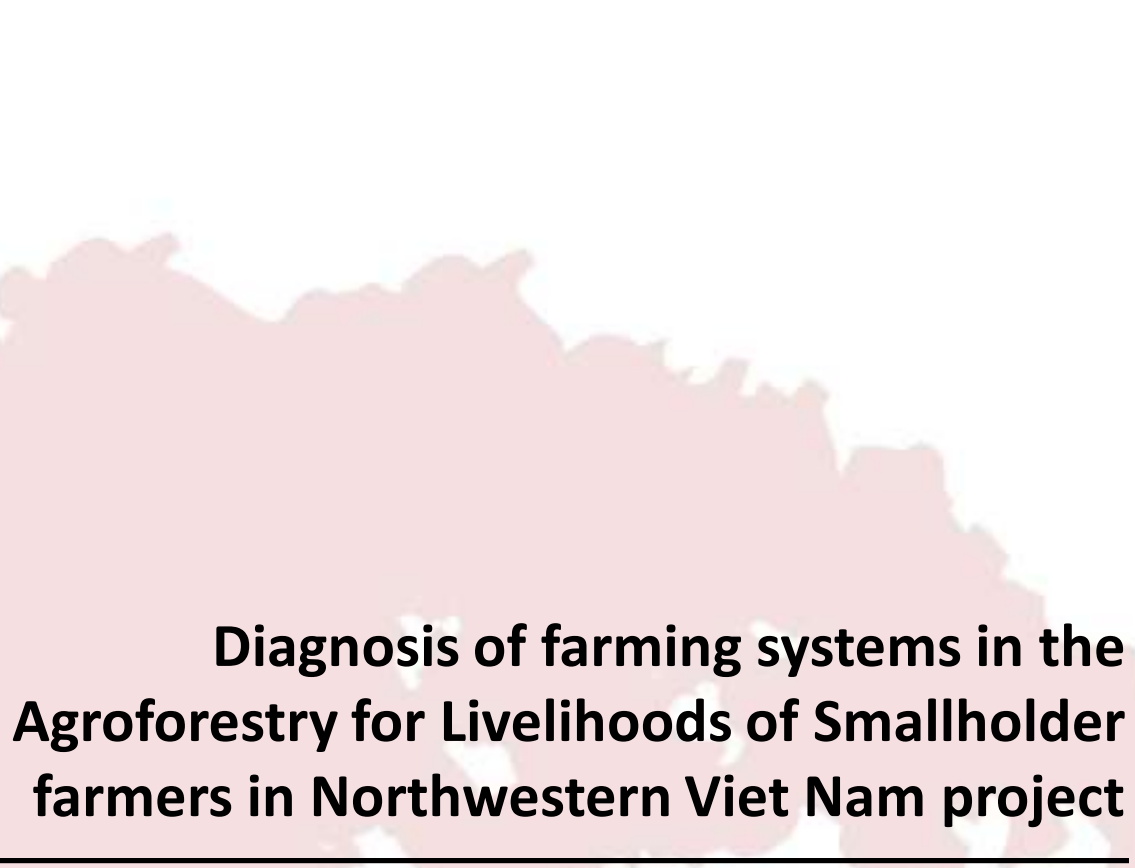

Hoang Thi Lua, Elisabeth Simelton, Ha Van Tiep, Vu Duc Toan, Nguyen Thi Hoa, Nguyen Van Chung, Phung Quoc Tuan Anh 



\section{Diagnosis of farming systems in the Agroforestry for Livelihoods of Smallholder farmers in Northwestern Viet Nam project}

Hoang Thi Lua, Elisabeth Simelton, Ha Van Tiep, Vu Duc Toan, Nguyen Thi Hoa, Nguyen Van Chung, Phung Quoc Tuan Anh 


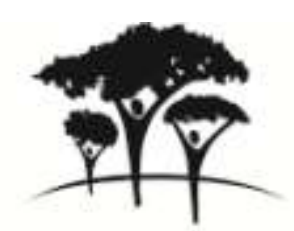

World Agroforestry Centre

TRANSFORMING LIVES AND LANDSCAPES

\section{Correct citation:}

Hoang TL, Simelton E, Ha VT, Vu DT, Nguyen TH, Nguyen VC, Phung QTA. Diagnosis of farming systems in the Agroforestry for Livelihoods of Smallholder farmers in Northwestern Viet Nam project. Working Paper no.161. Hanoi, Viet Nam: World Agroforestry Centre (ICRAF) Southeast Asia Regional Program. 24p. DOI: 10.5716/WP13033.PDF

This research project was carried out by the World Agroforestry Centre and local partners as part of the Agroforestry for Smallholders' Livelihoods in Northwestern Viet Nam project, funded by the Australian Centre for International Agricultural Research and the CGIAR Research Program on Forests, Trees and Agroforestry's component on Smallholders' Production Systems and Markets.

Titles in the Working Paper series disseminate interim results on agroforestry research and practices to stimulate feedback from the scientific community. Other publication series from the World Agroforestry Centre include agroforestry perspectives, technical manuals and occasional papers.

Published by the World Agroforestry Centre Viet Nam

No. 8, Lot 13A, Trung Hòa Street, Yen Hoa Ward, Cau Giay District, Hanoi, Viet Nam

Tel: +84437834644

Fax: +84 437834645

Email: icraf-vietnam@cgiar.org

Website: http://worldagroforestrycentre.org/regions/southeast_asia/Viet Nam

(C) World Agroforestry Centre 2013

Working Paper 161

\section{Photos:}

The views expressed in this publication are those of the author(s) and not necessarily those of the World Agroforestry Centre.

Articles appearing in this publication may be quoted or reproduced without charge, provided the source is acknowledged.

All images remain the sole property of their source and may not be used for any purpose without written permission of the source. 


\section{About the authors}

Hoang Thi Lua is a researcher with the World Agroforestry Centre Viet Nam and is the field coordinator of the Agroforestry for Smallholders' Livelihoods in Northwestern Viet Nam, funded by the Australian Centre for International Agricultural Research and the CGIAR Research Program on Forests, Trees and Agroforestry's component on Smallholders' Production Systems and Markets. She holds a doctorate in Agriculture and Horticulture Science from Germany (2003) and has more than seven years' experience in different projects in the fields of forestry, poverty reduction and sustainable natural resources management.

Elisabeth Simelton is a researcher with the World Agroforestry Centre VietNam. Her main research interests cover climate-change impacts and adaptation, food security and multifunctional landscapes, in particular, in Southeast and East Asia and Southern Africa. She was co-author of the Agroforestry for Smallholders' Livelihoods in Northwestern Viet Nam proposal and is now involved in agroforestry policy research and training extension workers in the project. She obtained her doctorate in Geography in 2007 on climate and human impacts on land use in Northern China and have over ten years experience in agricultural and rural development.

Ha Van Tiep is a vice director of the North-West Forestry Science and Production Centre in Son La province, which is part of the Forestry Science Institute of Viet Nam. He holds a master of science in Natural Resource Management from the Asian Institute of Technology, Thailand (2007). He is a $\mathrm{PhD}$ candidate at Utrecht University in the Netherlands and has 16 years' experience in sustainable use of natural resources, plantation forests, agroforestry, community forest management and sustainable livelihoods of local farmers.

Vu Duc Toan is a lecturer and head of the Experiment Division, Agroforestry Department, Tay Bac University. He is a graduate in Silviculture from Tay Bac University and will soon receive a master degree in Forestry from the Viet Nam Forestry University. He has four years' experience in sociological research. He participated in the evaluation of the project on sustainable upland farming in Moc Chau and in the assessment of the social impacts of the advanced cooking stove project, both in Son La province. 
Nguyen Thi Hoa is a researcher with the World Agroforestry Centre Viet Nam. Currently, she is involved in monitoring and evaluation of the Agroforestry for Smallholders' Livelihoods in Northwestern Viet Nam project. She obtained a MSc in Environment and Natural Resource Management in Thailand in 2009 and has three year of experience in forestry and natural resource management.

Phung Quoc Tuan Anh is head of the Division of Technology Transfer and Development, Tay Bac Agroforestry Research and Development Centre. Anh has a university diploma in Forest Resources and Environmental Management and earned a master degree in Farming at Thai Nguyen University of Agroforestry. He has six years' research experience in northwestern Viet Nam in such areas as sustainable upland cultivation; research on, and development of, local farming practices; studies on rubber trees and associated crops in plantations during the establishment phase.

Nguyen Van Chung is a researcher with the Agroforestry Division, Northern Mountainous Agriculture and Forestry Institute. Earning a master degree in Forestry in 2010, Chung has seven years' research experience in agroforestry in the northern areas. He has joined various areas of research and technology transfer, including upland sustainable farming; sustainable economic development of planted forests; biodiversity conservation; rubber tree development for the northern mountainous regions and other projects with international partners. 


\section{Abstract}

The ‘Agroforestry for Livelihoods of Smallholder farmers in Northwestern Viet Nam' project (20112016) focuses on agroforestry trials on farms in the northwestern mountainous region of the country. The objective of the study was to make an inventory of the current upland farming systems and identify key strengths and weaknesses of each system, including economic efficiency. The farming system diagnosis was based on participatory assessments and focus group discussions and in-depth interviews with 45 project 'farmer co-operators' in 17 villages in Yen Bai, Son La and Dien Bien provinces. The study covered three agro-ecozones and four ethnic groups: $<600$ masl, generally populated by Kinh (two villages); 600-800 masl, dominated by Thai (six villages) and > 800 masl, including H'mong and Kho Mu (nine villages). The economic assessment accounts for annual inputs and labor costs. To our knowledge, there was no previous systematic study at this scale. The inventory identified over 20 different farming systems. However, regardless of agro-ecozones and ethnicity, the predominant land-use on upland slopes was mono-cultivation of staple crops. Over $90 \%$ of the farmer co-operators grew maize, which was also the main source of income for $82 \%$. The estimated economic profit of this system ranged from below VND 1 million to 12.5 million per hectare per year. The main drawback of this system was declining soil fertility and yields. In addition, upland farmers' profits were generally low, ranging from VND 2.4 million (maize) through VND 20 million (cassava with shan tea) to VND 26 million (tomato) per hectare per year. Profits were lower at higher elevations. This was because 1) in response to declining soil fertility, increased amounts of fertilizers were applied but the productivity failed to compensate for increasing production costs; 2) local varieties of hill rice and maize seem to have degenerated; 3) farmers sold unprocessed grains and tubers at low, and volatile, prices because their market access and storage capacity was limited; and 4) the estimated labour costs in this study might be overestimated for distant fields. Farmers, therefore, wanted to find alternative farming systems, however, few were aware of appropriate tree-based conservation farming practices. Certain types of agroforestry existed on a small scale, for example, home gardens with fruit trees or coffee with timber trees or fruit trees as shade (in Son La), cassava intercropped with shan tea (in Yen Bai), cardamom under forest canopy (in Dien Bien). This study identified several potential agroforestry models, for example, a combination of staple crops for shortterm income, grass strips for protection from soil erosion and feed, and trees for medium-to-longerterm income. The findings of the study will help identify agroforestry systems with potential for wider 
adoption and will lay the groundwork for the design or redesign of effective agroforestry research in the region.

Keywords: Farming system diagnosis, monocropping, agroforestry, Northwest Vietham, farmers' adoption 


\section{Acknowledgements}

We acknowledge the valuable contribution of:

- Department for Agriculture and Rural Development in Son La, Dien Bien and Yen Bai provinces for their participation and support during the survey;

- research staff of the Northern Mountainous Agriculture and Forestry Institute, North-West Forestry Science and Production Center and Tay Bac University for their active participation and logistics; and

- Dr Delia Catacutan for her valuable technical support during the design and implementation of the survey and advice during the completion of this report.

The project is funded by the Australian Centre for International Agricultural Research and the CGIAR Research Program on Forests, Trees and Agroforestry's component on Smallholders' Production Systems and Markets. 



\section{Contents}

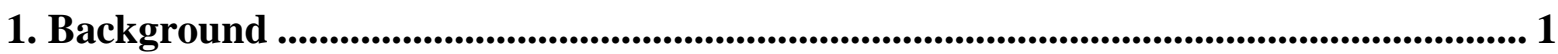

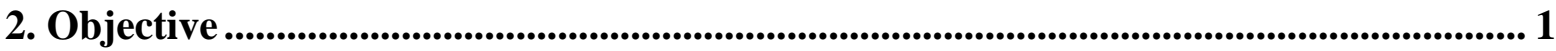

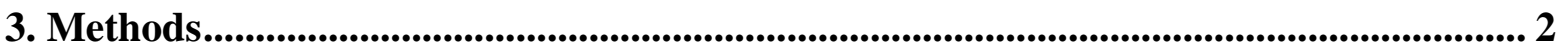

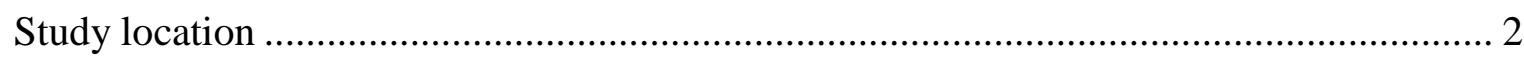

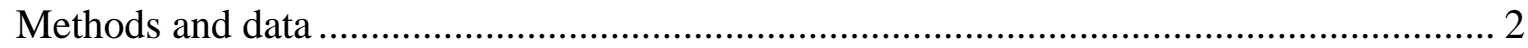

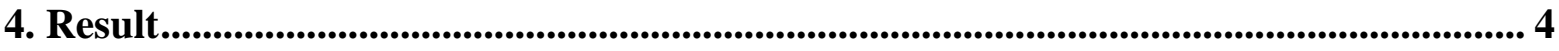

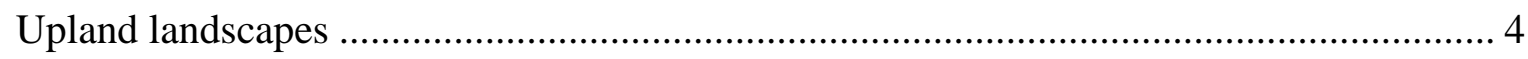

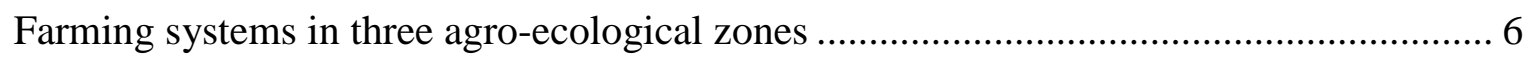

Economic effectiveness of some dominant farming systems in the study villages ........... 15

Potential plant species by agro-ecological zone ....................................................... 17

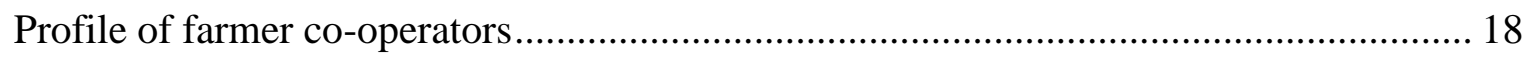

5. Discussion and conclusion .................................................................................................... 22

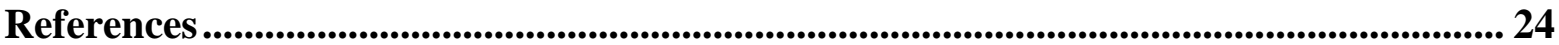

\section{List of Figure}

Figure 1. Participatory village transect map ................................................................. 3

Figure 2. Focus group discussion, Che A village ............................................................. 3

Figure 3. Areas of maize cultivation, Che A village (> 800 masl) .................................... 12

Figure 4. Home garden in Ta Phin/Tua Chua (> 800 masl)............................................ 12

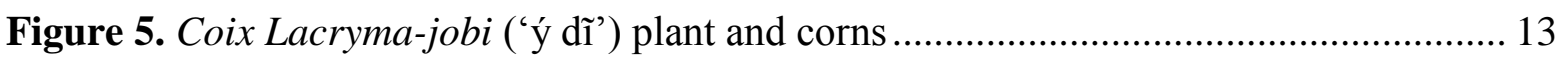

Figure 6. Traditional agroforestry system, forest-terraced field and home garden in

Ta Xua, Ban Cong, Tram Tau 14

Figure 7. Intercropped maize and beans by H'Mong people in Ta Phin, Tua Chua, Dien Bien

Figure 8. Crops provide the main income for households ................................................. 19

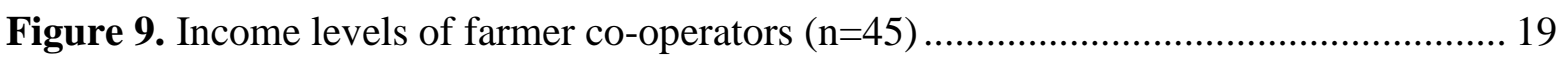

Figure 10. Most important factors in farmers' decisions on what trees and/or crops to plant $(\mathrm{n}=45)$

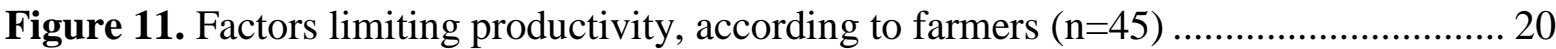




\section{List of Tables}

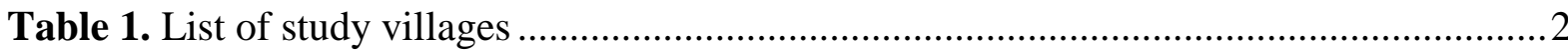

Table 2. Sample transect from a typical upland village, Nhop, in Thuan Chau district

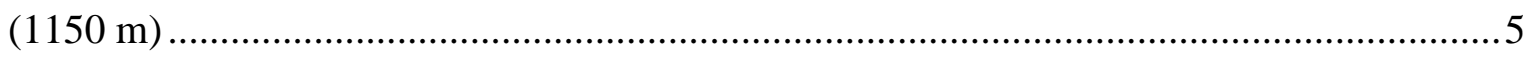

Table 3. Seasonal calendar of key species at the study sites ............................................. 7

Table 5. Economic effectiveness of selected farming systems ............................................ 16

Table 6. Species with high potential for agroforestry by agro-ecological zone...................... 18 


\section{Background}

The northwestern uplands of Viet Nam are characterized by a sub-humid, tropical climate with frost at high altitudes, and many remote and culturally diverse communities. Agriculture is predominantly self-subsistent with paddy in the valleys and maize, upland rice and cassava on the slopes. Maize is the most important food and fodder crop for most ethnic groups in this region (Hoang 2010).

However, these farming practices are unsustainable land uses that form a vicious circle of shorter crop cycles, no fallow and no protective soil cover during the onset of the rainy season, leading to soil erosion, declining yields and unstable livelihoods. Water scarcity further aggravates the difficulty of sustaining crop productivity and incomes.

Starting in 2011, the research project, Agroforestry for Smallholders' Livelihoods in Northwest Viet Nam (AFLI), seeks to improve the performance of smallholders' farming systems through agroforestry. The goal of the project is to establish more diverse and sustainable production systems and better income from tree products (Hoang 2011).

In 2012, after consultation with local farmers and researchers, the project established on-farm trials of improved agroforestry systems: maize intercropped with late fruiting longan; maize with 'son tra' (Docynia indica); 'shan' tea (Camellia sinensis var Shan) with fodder grasses; son tra with fodder grasses; macadamia with coffee and beans; and Amomum under shade.

To gain a deeper insight into the economic and environmental limitations of existing farming systems and to validate the suitability of the trial agroforestry systems (for example, the selection of plant species and the levels of farming intensification, as well as gather data for a planned impact assessment), a diagnosis of farming systems was carried out in the 17 research villages in Son La, Dien Bien and Yen Bai provinces in May 2012. The diagnosis involved 45 farmers participating in the project in 2012, from here on referred to as 'farmer co-operators'.

\section{Objective}

The overall objective of the study was to document the existing farming systems at the project's sites to further inform the design of the agroforestry research trials and assess the scalability of the tested agroforestry systems. There were five specific objectives.

1. To identify the prevailing farming systems, including current agroforestry systems where available, in the three agro-ecological zones of the project's areas and make a rapid participatory assessment of their economic and environmental effectiveness.

2. To assess the potential economic benefits, as well as the associated risks, of proposed agroforestry technologies.

3. To identify alternatives to the fodder shrubs and tree species previously selected for the on-farm trials. The identified species should be economically viable, address soil erosion and soil fertility issues, and have potential for widespread planting in the northwestern region.

4. To confirm or adjust the designs of the agroforestry trials and generate recommendations for improvement, that is, additional trials or treatments. The study should also advise on the scalability of the improved agroforestry systems.

5. To collect data on the farming practices of the farmer co-operators. 


\section{Methods}

\section{Study location}

The study was conducted in 17 villages in 11 communes of six districts in the provinces of Yen Bai (five villages), Son La (seven villages) and Dien Bien (five villages) (table 1) that had on-farm agroforestry trials in the AFLI project.

The villages were located between 300 and 1250 masl. The number of households in each village ranged 55-200. H'mong and Kho Mu people lived in nine villages located at high elevations above 800 masl; Thai people in another six villages at intermediate elevations of 600-800 masl; and Kinh people in two villages at low elevations below 600 masl. Table 1 shows the locations of the villages.

Table 1. List of study villages

\begin{tabular}{|c|c|c|c|c|c|c|}
\hline District, Province & Commune & Village & \multicolumn{2}{|c|}{$\begin{array}{c}\text { Elevation (masl) (agro- } \\
\text { ecozones) }\end{array}$} & $\begin{array}{l}\text { Number of } \\
\text { households }\end{array}$ & Ethnic group \\
\hline \multirow[t]{2}{*}{ Van Chan, Yen Bai } & Son Thinh & Hong Son & \multirow{2}{*}{$<600$} & 295 & 200 & Kinh \\
\hline & Son Thinh & Van Thi 4 & & 327 & 117 & Kinh \\
\hline \multirow[t]{4}{*}{ Mai Son, Son La } & Chieng Chan & Sai Luong & \multirow{4}{*}{$\begin{array}{c}600- \\
800\end{array}$} & 650 & 129 & Thai \\
\hline & Chieng Chan & $\mathrm{Na}$ Phuong & & 750 & 95 & Thai \\
\hline & Co Noi & Mon & & 600 & 152 & Thai \\
\hline & Co Noi & Phieng $\mathrm{Hy}$ & & 720 & 45 & H'mong \\
\hline \multirow[t]{2}{*}{ Tuan Giao, Dien Bien } & Quai Nua & Chan & \multirow{2}{*}{$\begin{array}{c}600- \\
800\end{array}$} & 650 & 48 & Thai \\
\hline & Quai Nua & Cha & & $600-800$ & 68 & Thai \\
\hline \multirow[t]{3}{*}{ Thuan Chau, Son La } & Chieng Bom & Nhop & \multirow[t]{3}{*}{$>800$} & 1150 & 84 & Thai \\
\hline & $\mathrm{Co} \mathrm{Ma}$ & $\mathrm{Co} \mathrm{Ma}$ & & 1150 & 66 & H'mong \\
\hline & Long $\mathrm{He}$ & Nong Coc $\mathrm{A}$ & & $1100-1250$ & 80 & Kho Mu \\
\hline \multirow[t]{2}{*}{ Tuan Giao, Dien Bien } & Toa Tinh & Che A & \multirow[t]{2}{*}{$>800$} & $1100-1250$ & 55 & H'mong \\
\hline & Toa Tinh & Hua Sa A & & $1000-1250$ & 80 & H'mong \\
\hline Tua Chua, Dien Bien & Ta Phin & Ta Phin & $>800$ & 1100 & 55 & H'mong \\
\hline \multirow[t]{2}{*}{ Van Chan, Yen Bai } & Suoi Giang & Giang B & \multirow[t]{2}{*}{$>800$} & $1100-1250$ & 79 & H'mong \\
\hline & Suoi Giang & Pang Cang & & $1100-1250$ & 116 & H'mong \\
\hline Tram Tau, Yen Bai & Ban Cong & Ta Xua & $>800$ & 1180 & 82 & H Mong \\
\hline
\end{tabular}

\section{Methods and data}

The diagnosis was conducted in four steps at each of the 17 villages.

1. Agro-ecological transect maps were created through village transect walks with 15 residents each. Dominant land-use systems associated with different elevation intervals and degrees of slope were identified, including current crop varieties, cropping systems and patterns (mono-cropping or crop association). Biophysical indicators were registered, such as soil type, erosion status and water resources. 


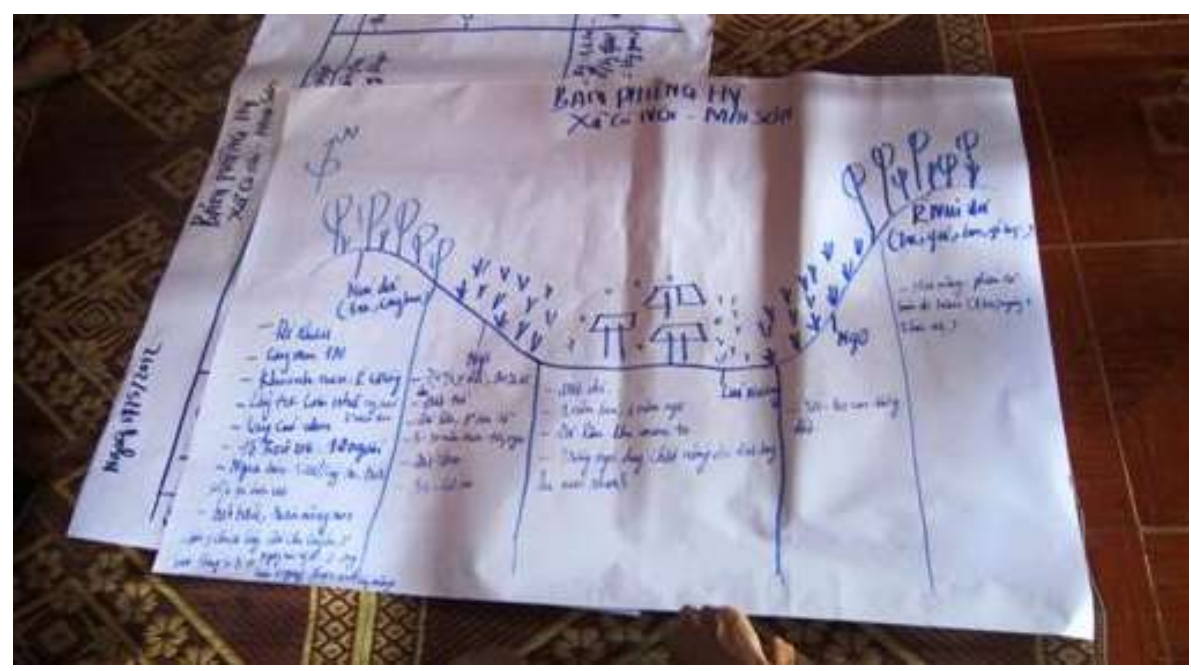

Figure 1. Participatory village transect map

2. Focus group discussions were conducted with 15 farmers per village, who were selected randomly by the team from a list of households. The discussions consisted of semi-structured interviews about the village's farming systems, including the farming calendar and an analysis of the strengths, weaknesses, opportunities and threats to the farming and agroforestry systems. The purpose was to generate information for a rapid economic assessment of the dominant cropping systems. The discussions helped the research team better understand the nature of the challenges faced by farmers in their farming practices, provided data on indigenous methods for preventing erosion and indicated the levels of farmers' awareness of agroforestry.

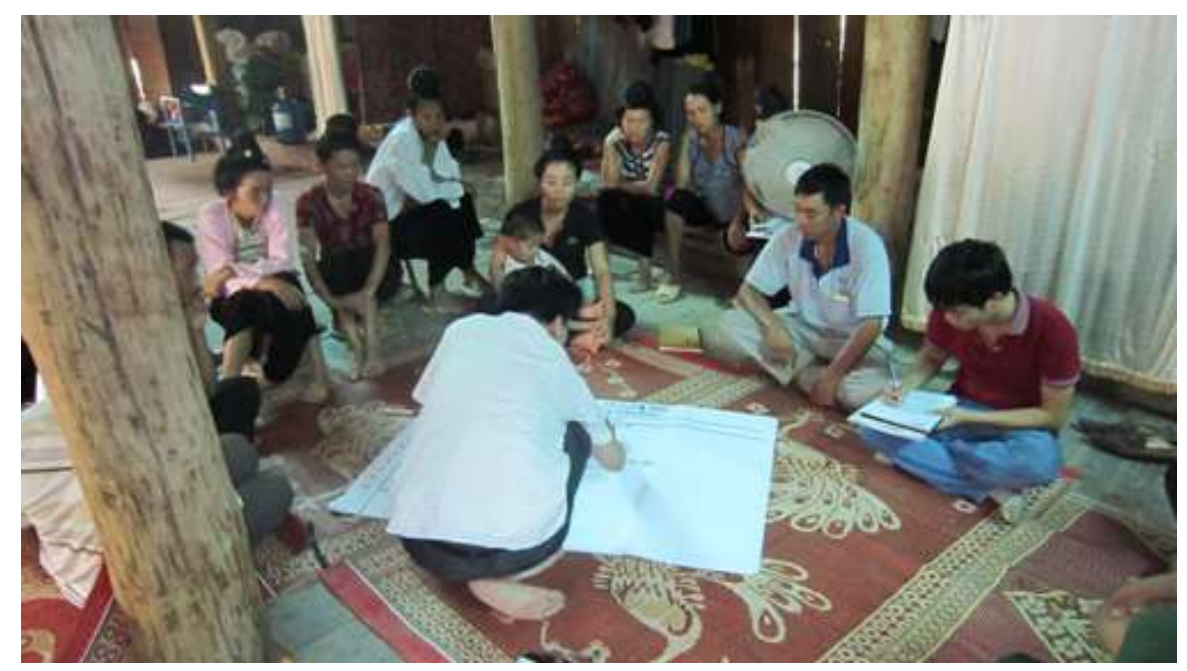

Figure 2. Focus group discussion, Che A village

3. The profit of the farming system per year was calculated as follows:

$$
\text { Total profit: } \mathbf{P}=\mathbf{T n}-\mathbf{C p}
$$

[equation 1]

Where $\mathrm{P}$ denotes profit (VND/ha/year), Tn refers to the total income (turnover) (VND/year) and $\mathrm{Cp}$ is the total cost for all inputs including labor time, seeds/seedlings, fertilizer, pesticides, herbicides, tools etc. (VND/year). 
The resulting economic effectiveness of the dominant farming systems was calculated as the average value of all inputs of the most recent harvest season; this information being obtained from the interviews with farmers. In reality, the figure varied by household owing to various factors, for example, soil quality, slope, investments (fertilizer) and distance from home to the planted area.

All inputs and outcomes were considered to be independent and not influenced by time (that is, static). Selling price was counted as the average product price of the latest harvesting season and the price of inputs was the price at the time of purchase. The cost of labour was set at VND 100.000/day.

For systems with perennial crops, such as forestry plantations (intercropping of non-timber forest product (NTFP) crops under the forest canopy), the economic effectiveness was based on the latest harvest using the same principle as above. From this calculation, the effectiveness of the system at the first harvest will be negative or very low in comparison to that of subsequent years, owing to high initial cost of system establishment.

4. Household surveys were conducted as structured questionnaires with 45 farmer co-operators. The survey aimed to collect baseline information for an impact assessment of the project later (questionnaire is provided in annex 1).

\section{Result}

\section{Upland landscapes}

Combining the 17 transects shows some common characteristics (the transects for each village are provided in annex 2-18).

As an example, table 2 shows a transect of Nhop village, Chieng Bom commune, dissected into typical land uses associated with certain topographical features and the status of soil, water and erosion, while the last row provides potential solutions identified by the farmers.

The highest point of a transect was usually rocky mountains with natural forests, descending along steep-to-moderate slopes with mono-cultivation of maize (staple crop) through comparatively flat areas with home gardens to paddy rice on the valley floor.

The quality of natural forests was generally low after years of overexploitation. Timber for housing was no longer available and NTFPs were very limited. Few farmers received benefits from their forests besides some firewood, medicinal plants and mushrooms. Farmers wanted to plant fastgrowing trees for timber for building material. 
Table 2. Sample transect from a typical upland village, Nhop, in Thuan Chau district (1150 m)

\begin{tabular}{|c|c|c|c|c|c|c|c|}
\hline & & moseneside & 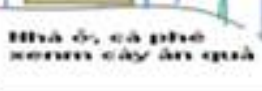 & 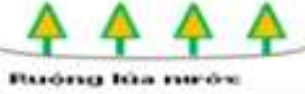 & 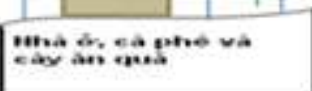 & 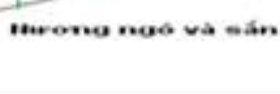 & \\
\hline Land use & $\begin{array}{l}\text { Plantation } \\
\text { and } \\
\text { regenerated } \\
\text { forest }\end{array}$ & $\begin{array}{l}\text { Cultivated } \\
\text { area (upland } \\
\text { crops) }\end{array}$ & Home garden & Paddy field & Home garden & $\begin{array}{l}\text { Cultivated area } \\
\text { (upland crops) }\end{array}$ & $\begin{array}{l}\text { Plantation and } \\
\text { regenerated } \\
\text { forest }\end{array}$ \\
\hline Slope & $\begin{array}{l}\text { Steep } \\
\left(>15^{\circ}\right)\end{array}$ & $\begin{array}{l}\text { Moderate } \\
\text { steep }\left(5-15^{\circ}\right)\end{array}$ & Flat $\left(<5^{\circ}\right)$ & Flat & Flat & $\begin{array}{l}\text { Moderate steep (5- } \\
\left.15^{\circ}\right)\end{array}$ & $\begin{array}{l}\text { Steep } \\
\left(>15^{\circ}\right)\end{array}$ \\
\hline Species & $\begin{array}{l}\text { Acacia } \\
\text { auriculiformis, } \\
\text { regenerated } \\
\text { shrubs }\end{array}$ & Cassava & $\begin{array}{l}\text { Canarium, coffee, } \\
\text { fruit species around } \\
\text { home garden }\end{array}$ & Paddy rice & $\begin{array}{l}\text { Canarium, coffee, fruit } \\
\text { species around home } \\
\text { garden }\end{array}$ & Cassava & $\begin{array}{l}\text { Bamboo, Acacia } \\
\text { auriculiformis, } \\
\text { regenerated } \\
\text { shrubs }\end{array}$ \\
\hline Soil status & $\begin{array}{l}\text { Rich soil } \\
\text { mixed with } \\
\text { gravel, black } \\
\text { colour, red } \\
\text { layer beneath. }\end{array}$ & $\begin{array}{l}\text { Red soil, no } \\
\text { gravel. }\end{array}$ & $\begin{array}{l}\text { Rich soil, flat with } \\
\text { high content humus. }\end{array}$ & Rich soil & $\begin{array}{l}\text { Rich soil, high content } \\
\text { of humus. }\end{array}$ & Red soil, no gravel & $\begin{array}{l}\text { Rich soil mixed } \\
\text { with little gravel; } \\
\text { black soil, red layer } \\
\text { beneath. }\end{array}$ \\
\hline Soil layer & $20-30 \mathrm{~cm}$ & $30-40 \mathrm{~cm}$ & $50-60 \mathrm{~cm}$ & $50-60 \mathrm{~cm}$ & $50-60 \mathrm{~cm}$ & $30-40 \mathrm{~cm}$ & $20-30 \mathrm{~cm}$ \\
\hline Difficulties & $\begin{array}{l}\text { Forest quality } \\
\text { low }\end{array}$ & $\begin{array}{l}\text { Water scarcity, } \\
\text { high soil } \\
\text { erosion }\end{array}$ & & Limited area for paddy & & $\begin{array}{l}\text { Water scarcity, high } \\
\text { soil erosion and } \\
\text { runoff }\end{array}$ & Forest quality low \\
\hline Potential Solutions & $\begin{array}{l}\text { Forest } \\
\text { regeneration } \\
\text { and } \\
\text { enrichment } \\
\text { with hybrid } \\
\text { acacia }\end{array}$ & $\begin{array}{l}\text { Tree-based } \\
\text { farming system }\end{array}$ & $\begin{array}{l}\text { Intercropping timber } \\
\text { or fruit tree species } \\
\text { and coffee }\end{array}$ & $\begin{array}{l}\text { Build irrigation system; } \\
\text { add manure }\end{array}$ & $\begin{array}{l}\text { Intercropping timber } \\
\text { or fruit tree species } \\
\text { and coffee }\end{array}$ & $\begin{array}{l}\text { Tree-based farming } \\
\text { system }\end{array}$ & $\begin{array}{l}\text { Forest } \\
\text { regeneration and } \\
\text { enrichment with } \\
\text { hybrid acacia }\end{array}$ \\
\hline
\end{tabular}


Water scarcity was common in nearly all villages. This affected the productivity of crops in several ways. First, nearly all agricultural production, especially on slopes, was rainfed. Second, land degradation lead to reduced waterholding capacity of the soils, thereby accentuating the water deficiency. Third, owing to water shortages, farmers could plant only one (autumn) crop a year while the fields were left without groundcover for months and exposed to early rains that accelerated the erosion process.

Farmers commonly said that on slopes the upper soil layer was becoming thinner over the years owing to intensive mono-cultivation: the soil is left bare during the months that have the most intense rainfall. This has resulted in continuously declining yields. In response, only one of the 17 villages (Phieng Hy) had measures to prevent soil erosion, including pipes to divert water and stone fences. Of the 45 farmer co-operators interviewed only one (a village leader) used soil-erosion prevention measures. Although a majority of the interviewed farmers were aware that soil degradation caused crop yields to decrease, none had consulted someone (for example, an extension worker) for a solution.

\section{Farming systems in three agro-ecological zones}

\section{Farming calendar}

Table 3 summarizes a farming calendar for key upland crops by elevation and province. Staple crops (maize, hill rice and cassava) were grown in all three agro-ecological zones from April through October/November, while peanuts and beans were both intercropped with the main crops and in rotation with them. For maize and hill rice, planting at high elevations started one month later than at lower elevations and harvests were up to two months later.

Paddy rice was grown at all elevations where water was available, however, only two villages (Tram Tau and Tuan Giao) had sufficient water for two crops per year. Single paddy crops grew from June to September and double crops were planted between February and April, harvested in July, and then planted again from August to November.

The dominant cash crops were tea in Yen Bai and coffee in Son La and Dien Bien. Shan tea was planted in late spring and early summer (May-July in Yen Bai and one month earlier in Dien Bien) and bush tea was planted in the autumn (August-October in Yen Bai). Coffee was planted during spring (for example, March-April in Mai Son, 600-800 masl, and one month later in Tuan Giao). Sugar cane was grown above 600 masl (Mai Son), planted in January/February and harvested in September/October on average three times during three consecutive years. The sugar cane yield declined after three harvests and needed to be replanted.

Tree species were generally planted between May and July in all three provinces. 
Table 3. Seasonal calendar of key species at the study sites

\begin{tabular}{|c|c|c|c|c|c|c|c|c|c|c|c|c|c|c|c|c|c|}
\hline \multicolumn{2}{|c|}{ Location } & \multirow{2}{*}{$\begin{array}{c}\text { Elevation } \\
\text { (masl) }\end{array}$} & \multirow{2}{*}{$\begin{array}{c}\text { Plant } \\
\text { species }\end{array}$} & \multirow{2}{*}{$\begin{array}{l}\text { No. of crop } \\
\text { seasons }\end{array}$} & \multirow[b]{2}{*}{ Jan } & \multirow[b]{2}{*}{ Feb } & \multirow[b]{2}{*}{ Mar } & \multirow[b]{2}{*}{ Apr } & \multirow[b]{2}{*}{ May } & \multirow[b]{2}{*}{ Jun } & \multirow[b]{2}{*}{ Jul } & \multirow[b]{2}{*}{ Aug } & \multirow[b]{2}{*}{ Sep } & \multirow[b]{2}{*}{ Oct } & \multirow[b]{2}{*}{ Nov } & \multirow[b]{2}{*}{ Dec } & \multirow[b]{2}{*}{ Jan } \\
\hline Province & District & & & & & & & & & & & & & & & & \\
\hline \multirow{12}{*}{ Son La } & \multirow{10}{*}{ Mai Son } & \multirow{10}{*}{$600-800$} & Maize & 1 & & & & & & & & & & & & & \\
\hline & & & Peanut & 1 & & & & & & & & & & & & & \\
\hline & & & \multirow{2}{*}{ Bean } & \multirow{2}{*}{2} & & & $\mathrm{C} 1$ & & & & & & & & & & \\
\hline & & & & & & & & & & & & & $\mathrm{C} 2$ & & & & \\
\hline & & & Hill rice & 1 & & & & & & & & & & & & & \\
\hline & & & Cassava & 1 & & & & & & & & & & & & & \\
\hline & & & Sugar cane & 1 & & & & & & & & & & & & & \\
\hline & & & Coffee & 1 & & & $\mathrm{P}$ & P & & & & & & & & & \\
\hline & & & Paddy & 1 & & & & & & & & & & & & & \\
\hline & & & Arrowroot & 1 & & & & & & & & & & & & & \\
\hline & \multirow{2}{*}{ Thuan Chau } & \multirow{2}{*}{$>800$} & Maize & 1 & & & & & & & & & & & & & \\
\hline & & & Hill rice & 1 & & & & & & & & & & & & & \\
\hline \multirow{13}{*}{ Dien Bien } & \multirow{11}{*}{ Tuan Giao } & \multirow{6}{*}{$<600$} & Maize & 1 & & & & $\mathrm{C} 1$ & & & $\mathrm{C} 2$ & & & & & & \\
\hline & & & Peanut & 2 & $\mathrm{C} 1$ & & & & & & & & $\mathrm{C} 2$ & & & & \\
\hline & & & Bean & 1 & & & & & & & & & & & & & \\
\hline & & & Hill rice & 1 & & & & & & & & & & & & & \\
\hline & & & Paddv rice & 2 & & & & $\mathrm{C} 1$ & & & & & & & & & \\
\hline & & & tenty & 2 & & & & & & & & $\mathrm{C} 2$ & & & & & \\
\hline & & & Maize & 1 & & & & & & & & & & & & & \\
\hline & & & Peanut & 1 & & & & & & & & & & & & & \\
\hline & & $>800$ & Hill rice & 1 & & & & & & & & & & & & & \\
\hline & & & cassava & 1 & & & & & & & & & & & & & \\
\hline & & & Coffee & 1 & & & & $\mathrm{P}$ & $\mathrm{P}$ & & & & & & & & \\
\hline & Ta Phin, Tua & $>800$ & Paddy rice & 1 & & & & & & & & & & & & & \\
\hline & Chua & $>000$ & Shan tea & 1 & & & & $P$ & $P$ & $P$ & & & & & & & \\
\hline
\end{tabular}




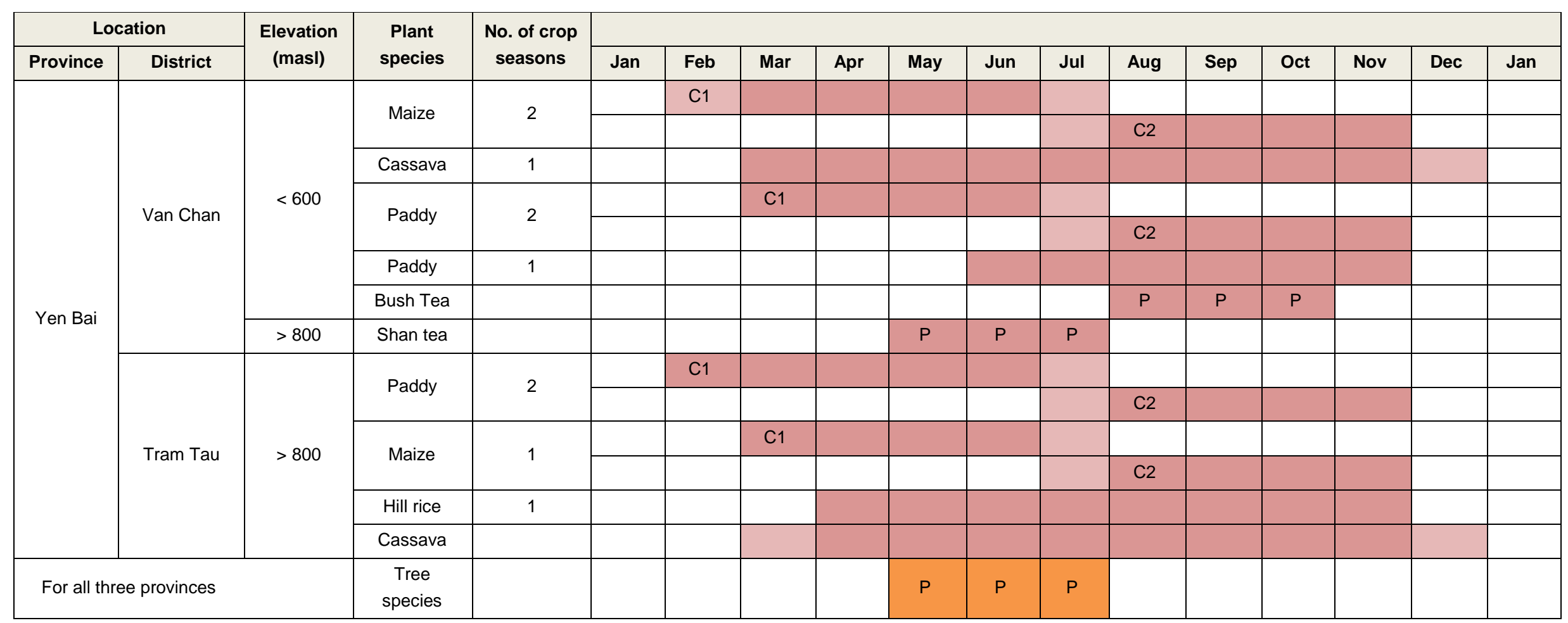




\section{Farming systems}

Table 4 below lists the dominant farming systems in the 17 villages according to the three elevation levels. Table 5 provides an economic analysis of the systems.

\section{Low elevations: $300-600$ masl}

The dominant farming system was mono-cultivation of staple crops on sloping land; the main species was hybrid maize with two harvests per year. Maize varieties were changed based on recommendations from the local agricultural extension service. The average yield ranged $3-$ $5 \mathrm{t} / \mathrm{ha} / \mathrm{crop}$.

The second most common crop was high-yield cassava, planted as monoculture where soil fertility had declined and the land could not support maize. The average yield ranged 18-22 t/ha/crop. In general, cassava cultivation brought low economic returns owing to low and unstable prices (table 5).

Paddy rice was planted as monoculture with two crops a year. The average yield ranged $3-5 \mathrm{t} / \mathrm{ha} / \mathrm{crop}$, depending on local conditions.

Bush tea was the leading cash crop, however, prolonged droughts had damaged plantations, affecting productivity.

Forest trees were mostly planted on heavily degraded soils unsuitable for agricultural crops. The most common species were fast-growing timber, such as acacia, hybrid eucalyptus, Manglietia and Styrax tonkinensis.

Home gardens contained scattered fruit tree species, for example, mango, longan, pomelo and orange combined with timber trees or vegetables. No improved agroforests were observed in the studied villages.

The focus groups identified several weaknesses or challenge, strengths and opportunities at this elevation.

Weaknesses or challenges

1. Inappropriate farming techniques, especially in farm management, post-harvest practices and processing of farm products.

2. Uneven quality of agricultural products owing to different input levels and varieties.

3. Low and unstable prices of agricultural produce as well as limited market access, resulting in low incomes and low investment in farm development.

Strengths

1. Kinh people accounted for over $90 \%$ of the population at this elevation. They were considered to be more advanced in adapting and accessing new farming technologies compared to others.

2. Available labour and traditionally hardworking.

3. Good knowledge of cultivating traditional and some newly imported species, such as hybrid eucalyptus and macadamia from Australia and fodder grass species.

4. Land was relatively flatter compared to other elevations.

5. Land and climate suitable for a wide range of crops. 


\section{Opportunities}

1. Good infrastructure in terms of transport, services and information exchange.

2. High-yielding and resistant crop varieties available.

3. Local people keen on adopting any farming innovation that can bring higher incomes.

4. Modern cultivation techniques available.

Table 4. Farming systems in the study villages, by elevation

\begin{tabular}{|c|c|c|c|c|}
\hline Elevation & Topography & Site & Ethnicity & Dominant farming systems \\
\hline \multirow[t]{4}{*}{$300-600 \mathrm{~m}$} & Gently sloping & $\begin{array}{l}\text { Van Thi } 4 \text { village, Son } \\
\text { Thinh commune, Van } \\
\text { Chan district, Yen Bai }\end{array}$ & Kinh & $\begin{array}{l}\text { - Maize monoculture } \\
\text { - Cassava monoculture } \\
\text { - Bush tea } \\
\text { - Acacia, eucalyptus hybrid } \\
\text { - Home fruit garden system }\end{array}$ \\
\hline & $\begin{array}{l}\text { Flat, irrigation } \\
\text { water available }\end{array}$ & $\begin{array}{l}\text { Hong Son village, Son } \\
\text { Thinh commune, Van } \\
\text { Chan district, Yen Bai }\end{array}$ & Kinh & $\begin{array}{l}\text { - Maize monoculture } \\
\text { - Cassava monoculture } \\
\text { - Purple sugar cane } \\
\text { - Paddy rice, vegetables } \\
\text { - Home fruit garden system } \\
\text { - Plantation forest (acacia, eucalyptus) } \\
\end{array}$ \\
\hline & $\begin{array}{l}\text { Steep, rocky } \\
\text { slopes; water } \\
\text { scarcity }\end{array}$ & $\begin{array}{l}\text { Phieng Hy village, Mai } \\
\text { Son district, Son La } \\
\text { province }\end{array}$ & H'mong & $\begin{array}{l}\text { - Maize monoculture } \\
\text { - Beans or pumpkins after maize harvesting } \\
\text { - Upland rice monoculture } \\
\text { - Arrowroot (Canna edulis) monoculture } \\
\text { - Home fruit garden system }\end{array}$ \\
\hline & Flat land & $\begin{array}{l}\text { Mon village, Co Noi } \\
\text { commune, Mai Son } \\
\text { district, Son La } \\
\text { province }\end{array}$ & Thai & $\begin{array}{l}\text { - Maize monoculture } \\
\text { - Paddy rice monoculture } \\
\text { - Upland rice monoculture } \\
\text { - Sugar cane monoculture } \\
\text { - Cassava monoculture } \\
\text { - Beans intercropped with maize and cassava } \\
\text { - Coffee monoculture and intercropped in home } \\
\text { garden } \\
\text { - Home fruit garden (mango, grapefruit, longan) }\end{array}$ \\
\hline \multirow{4}{*}{$600-800 \mathrm{~m}$} & $\begin{array}{l}\text { Sloping land; } \\
\text { water scarcity }\end{array}$ & $\begin{array}{l}\text { Sai Luong village, } \\
\text { Chieng Chan } \\
\text { commune, Mai Son } \\
\text { district, Son La } \\
\text { province }\end{array}$ & Thai & $\begin{array}{l}\text { - Maize monoculture } \\
\text { - Maize rotated with beans (some households) } \\
\text { - Cassava monoculture } \\
\text { - Paddy rice (limited owing to lack of water) } \\
\text { - Newly established coffee plantation } \\
\text { - Eucalyptus }\end{array}$ \\
\hline & $\begin{array}{l}\text { Moderate slopes; } \\
\text { a bit rocky; water } \\
\text { scarcity }\end{array}$ & $\begin{array}{l}\text { Nhop village, Chieng } \\
\text { Bom, commune, Thuan } \\
\text { Chau district, Son La } \\
\text { province }\end{array}$ & Thai & $\begin{array}{l}\text { - Coffee intercropped with fruit trees, native } \\
\text { Canarium } \\
\text { - Cassava monoculture } \\
\text { - Upland rice monoculture } \\
\text { - Paddy rice: one crop/year } \\
\text { - Maize monoculture (very little) } \\
\text { - Eucalyptus hybrid }\end{array}$ \\
\hline & $\begin{array}{l}\text { Sloping land; } \\
\text { water scarcity }\end{array}$ & $\begin{array}{l}\text { Cha village, Quai Nua } \\
\text { commune, Tuan Giao } \\
\text { district, Dien Bien } \\
\text { province }\end{array}$ & Thai & $\begin{array}{l}\text { - Maize monoculture rotated with peanuts within } \\
\text { a year } \\
\text { - Cassava monoculture } \\
\text { - Paddy rice (one crop/year) in rotation with } \\
\text { peanuts after harvesting } \\
\text { - Newly established coffee plantation (two years } \\
\text { ago) } \\
\text { - Fruit trees: longan, plum, mango }\end{array}$ \\
\hline & $\begin{array}{l}\text { Flat land; water } \\
\text { available for paddy }\end{array}$ & $\begin{array}{l}\text { Chan village, Quai Nua } \\
\text { commune, Tuan Giao }\end{array}$ & Thai & $\begin{array}{l}\text { - Paddy rice: two crops/year } \\
\text { - Maize monoculture(rarely in rotation with }\end{array}$ \\
\hline
\end{tabular}




\begin{tabular}{|c|c|c|c|c|}
\hline & rice & $\begin{array}{l}\text { district, Dien Bien } \\
\text { province }\end{array}$ & & $\begin{array}{l}\text { beans and peanut) } \\
\text { - Upland rice in rotation with cassava and fallow } \\
\text { - Home fruit garden }\end{array}$ \\
\hline \multirow{8}{*}{$>800 \mathrm{~m}$} & $\begin{array}{l}\text { Sloping land; } \\
\text { water scarcity }\end{array}$ & $\begin{array}{l}\text { Nong Coc A, village, } \\
\text { Long He commune, } \\
\text { Thuan Chau district, } \\
\text { Son La province }\end{array}$ & Kho Mu & $\begin{array}{l}\text { - Upland rice rotated with cassava and fallow } \\
\text { - Son tra monoculture with high tree density } \\
\text { - Son tra and beans } \\
\text { - Upland rice intercropped with son tra (only one } \\
\text { household in the whole village) } \\
\text { - Arrowroot with son tra (only one household) }\end{array}$ \\
\hline & $\begin{array}{l}\text { Sloping land; } \\
\text { water scarcity }\end{array}$ & $\begin{array}{l}\text { Co Ma village, Co Ma } \\
\text { commune, Thuan Chau } \\
\text { district, Son La } \\
\text { province }\end{array}$ & H'mong & $\begin{array}{l}\text { - Maize intercropped with pumpkins } \\
\text { - Maize monoculture } \\
\text { - Upland rice rotated with cassava and fallow } \\
\text { - Upland rice intercropped with H'Mong } \\
\text { cucumber } \\
\text { - Terraced rice }\end{array}$ \\
\hline & $\begin{array}{l}\text { High steep sloping } \\
\text { land; natural forest } \\
\text { around the village }\end{array}$ & $\begin{array}{l}\text { Hua Sa A village, Toa } \\
\text { Tinh commune, Tuan } \\
\text { Giao district, Dien Bien } \\
\text { province }\end{array}$ & H'mong & $\begin{array}{l}\text { - Maize monoculture } \\
\text { - Upland rice monoculture } \\
\text { - Local cassava } \\
\text { - Coix lacryma-jobi monoculture } \\
\text { - Coffee monoculture plantation (planted in } \\
2010) \\
\text { - Son tra intercropped with upland rice or Pinus } \\
\text { - Scattered fruit trees: peach, plum, pear } \\
\text { - Pine, Vernicia montana } \\
\text { - Amomum under forest canopy }\end{array}$ \\
\hline & $\begin{array}{l}\text { Sloping land; } \\
\text { water scarcity }\end{array}$ & $\begin{array}{l}\text { Che A village, Toa Tinh } \\
\text { commune, Tuan Giao } \\
\text { district, Dien Bien } \\
\text { province }\end{array}$ & H'mong & $\begin{array}{l}\text { - Maize monoculture (small areas intercropped } \\
\text { with beans or peanuts as subsistence crops) } \\
\text { - Upland rice rotated with cassava, fallow } \\
\text { - Upland rice intercropped with H'mong } \\
\text { cucumber } \\
\text { - Coix lacryma-jobi monoculture or intercropped } \\
\text { with H'mong cucumber }\end{array}$ \\
\hline & $\begin{array}{l}\text { High sloping and } \\
\text { rocky land }\end{array}$ & $\begin{array}{l}\text { Ta Phin } 2 \text { village, Ta } \\
\text { Phin commune, Tua } \\
\text { Chua district, Dien Bien } \\
\text { province }\end{array}$ & H'mong & $\begin{array}{l}\text { - Maize intercropped with beans and pumpkins } \\
\text { - Upland rice monoculture } \\
\text { - Terraced rice } \\
\text { - Shan tea } \\
\text { - Cardamom under natural forest canopy } \\
\text { - Scattered fruit trees: peach, plum, pear }\end{array}$ \\
\hline & $\begin{array}{l}\text { High rocky sloping } \\
\text { and }\end{array}$ & $\begin{array}{l}\text { Giang B village, Suoi } \\
\text { Giang commune, Van } \\
\text { Chan district, Yen Bai } \\
\text { province }\end{array}$ & H'mong & $\begin{array}{l}\text { - Maize monoculture } \\
\text { - Upland rice monoculture (low economic return) } \\
\text { - Paddy rice: one crop/year } \\
\text { - Cassava monoculture } \\
\text { - Shan tea } \\
\text { - Manglietia conifer (newly established two years } \\
\text { ago) } \\
\text { - Scattered fruit trees: peach, plum, pear }\end{array}$ \\
\hline & $\begin{array}{l}\text { Moderate slopes; } \\
\text { irrigation water } \\
\text { available }\end{array}$ & $\begin{array}{l}\text { Pang Cang village, } \\
\text { Suoi Giang commune, } \\
\text { Van Chan district, Yen } \\
\text { Bai province }\end{array}$ & H'mong & $\begin{array}{l}\text { - Maize intercropped with pumpkins } \\
\text { - Shan tea intercropped with cassava } \\
\text { - Paddy rice as subsistence crop }\end{array}$ \\
\hline & $\begin{array}{l}\text { Sloping land with } \\
\text { small proportion of } \\
\text { stone }\end{array}$ & $\begin{array}{l}\text { Ta Xua village, Ban } \\
\text { Cong commune, Tram } \\
\text { Tau district, Yen Bai } \\
\text { province. }\end{array}$ & H'mong & $\begin{array}{l}\text { - Maize intercropped with pumpkins } \\
\text { - Terraced rice: two crops/year } \\
\text { - Upland rice } \\
\text { - Cassava monoculture } \\
\text { - Coix lacryma-jobi monoculture } \\
\text { - Son tra } \\
\text { - Pine } \\
\text { - Scattered fruit trees: peach, plum, son tra }\end{array}$ \\
\hline
\end{tabular}




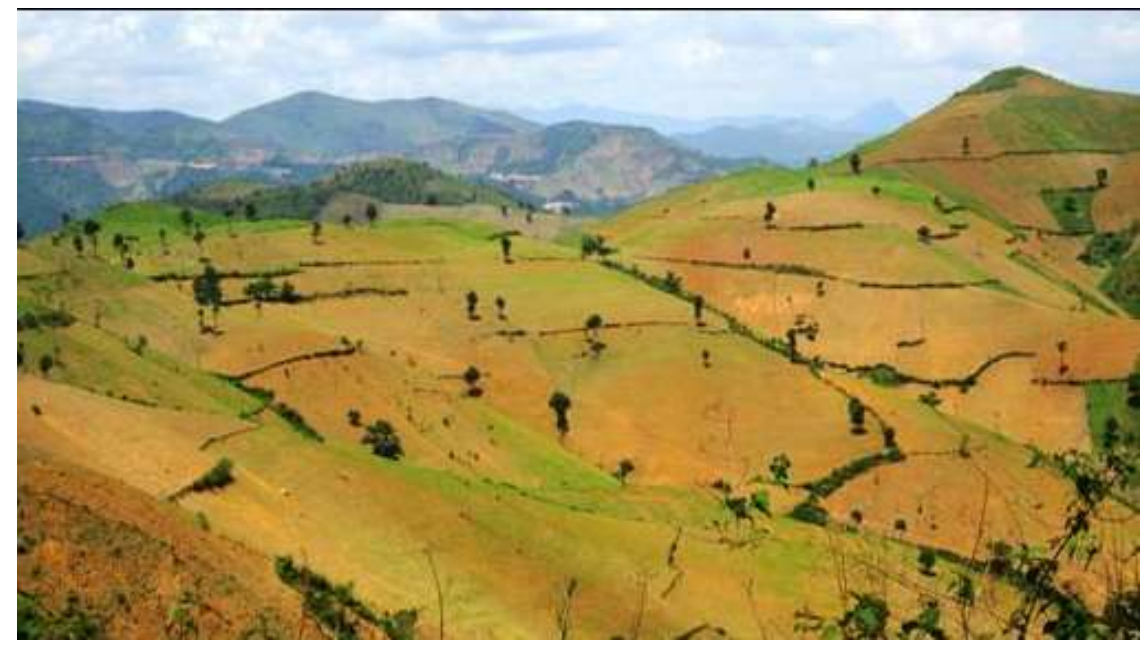

Figure 3. Areas of maize cultivation, Che A village (> 800 masl)

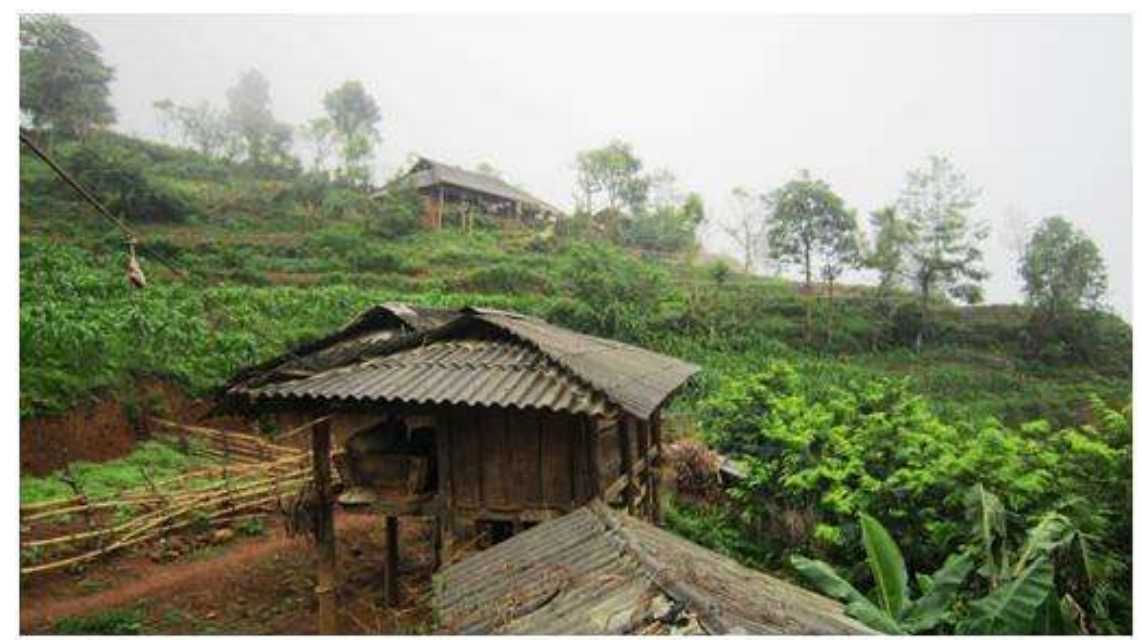

Figure 4. Home garden in Ta Phin/Tua Chua (> 800 masl)

\section{Intermediate elevations: 600-800 masl}

The dominant farming practices were mono-cultivation of food crops on sloping land, similar to those at elevation below 600 masl. In addition, in Mai Son district in Son La province there were a few small-scale agroforestry systems, such as mango or longan intercropped with food crops and coffee planted under fast-growing timber species.

Paddy rice cultivation was restricted owing to water scarcity. In areas without water for the second rice crop, peanuts were planted instead of rice.

Maize dominated both on slopes and lowlands. In some areas, maize was planted in rotation with peanuts or beans, giving one crop a year. The average yield of maize per crop was higher than that achieved at elevations below 600 masl, especially in Mai Son district where farmers planted hybrid 
maize varieties with high levels of fertilizers, for commercial purposes, such as DK 9901, CP 888, CP 333, NK 54, NK 6326, NK 67, NK 66, NK 6654. The yields reached up to 8 t/ha.

High-yield cassava was harvested annually, while native cassava was planted as perennial crop. Usually cassava was planted in rotation with upland rice or maize on degraded land.

Upland rice was planted as monoculture on hillsides in rotation with cassava. Despite yields being low (1.2-1.4 t/ha) and decreasing for years owing to depletion of soil nutrients, it was maintained as an important subsistence crop.

Monoculture coffee plantations had expanded over the last few years. Farmers preferred coffee to other traditional food crops because of its higher economic returns. Some common forest tree species like Eucalyptus and Canarium nigrum were planted in limited numbers. Fruit tree species in home gardens - such as longan, mango, plum and custard apple - were grown scattered through the gardens and as shade trees for coffee.

The key problems and challenges were similar to those at $<600$ masl. The main differences were owing to greater levels of slope and elevation, water scarcity and soil degradation; limited infrastructure; poor access to markets and services; and low investment capacity.

\section{High elevations: above 800 masl}

The farming systems at high elevations were less diverse in terms of species than at lower elevations, primarily consisting of monocultivated staple crops (maize or cassava) on sloping hillsides.

The crops grown by the H'mong people were mainly for subsistence. Compared with lower elevations, farmers at these levels used local varieties of hill rice, maize and cassava with less inputs and lower yields. The average maize yield varied 1.5-3 t/ha/year, cassava 10-15 t/ha/year and upland rice at most $1 \mathrm{t} / \mathrm{ha}$. Crop yields were said to be declining owing to germplasm degeneration. Some crop species were traditionally intercropped or planted in rotation, such as maize intercropped with pumpkins or beans, and upland rice with H'mong cucumber.

Paddy rice was mainly planted on terraces in a traditional agroforestry system: natural forest at the top of the hill followed by terraced rice fields and home gardens.

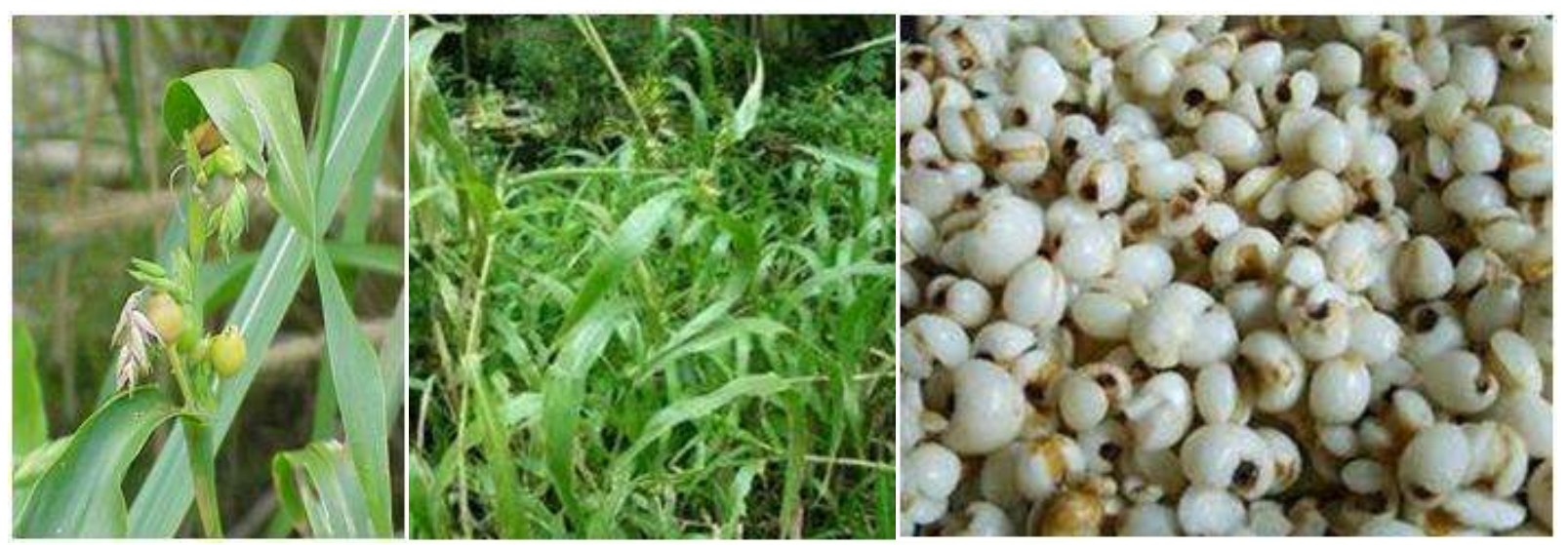

Figure 5. Coix Lacryma-jobi ('ý dĩ’) plant and corns 
Coix Lacryma-jobi ('ý dĩ') is a local staple crop that has been planted over a small area since a market is not yet available. However, Coix Lacryma-jobi is a potential crop for upland areas thanks to its drought tolerance and wind resistance. It can grow well in areas with low nutrient content that are no longer suitable for growing upland rice. Coix Lacryma-jobi has an average yield of 2.2-2.4 t/ha, twice as high as upland rice. It needs less tending while being resistant to pest and disease.

In Suoi Giang commune, some farmers started intercropping cassava with the existing shan tea plantation to improve the economic return of the system.

Forest tree species were mainly temperate species with long rotation, such as pine, Vernicia montana, Manglietia conifer, Fokienia hodginsii ('po mu'). In home gardens, common fruit trees were pear, plum and son tra. Some households planted son tra for additional income with the support of local reforestation projects.

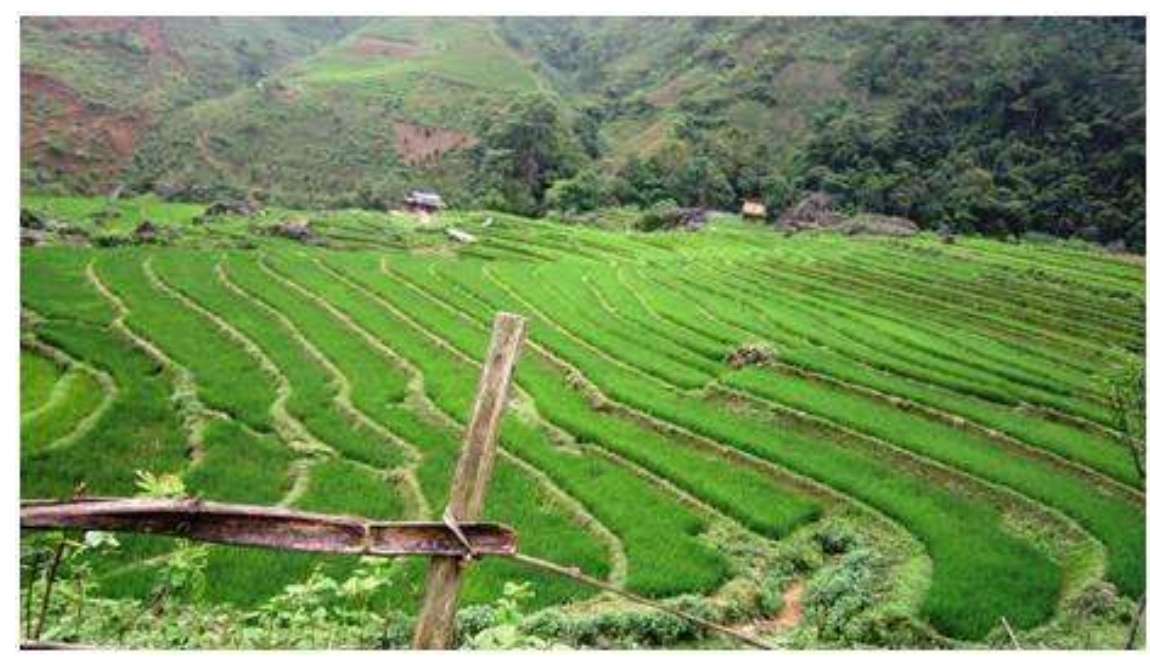

Figure 6. Traditional agroforestry system, forest-terraced field and home garden in Ta Xua, Ban Cong, Tram Tau

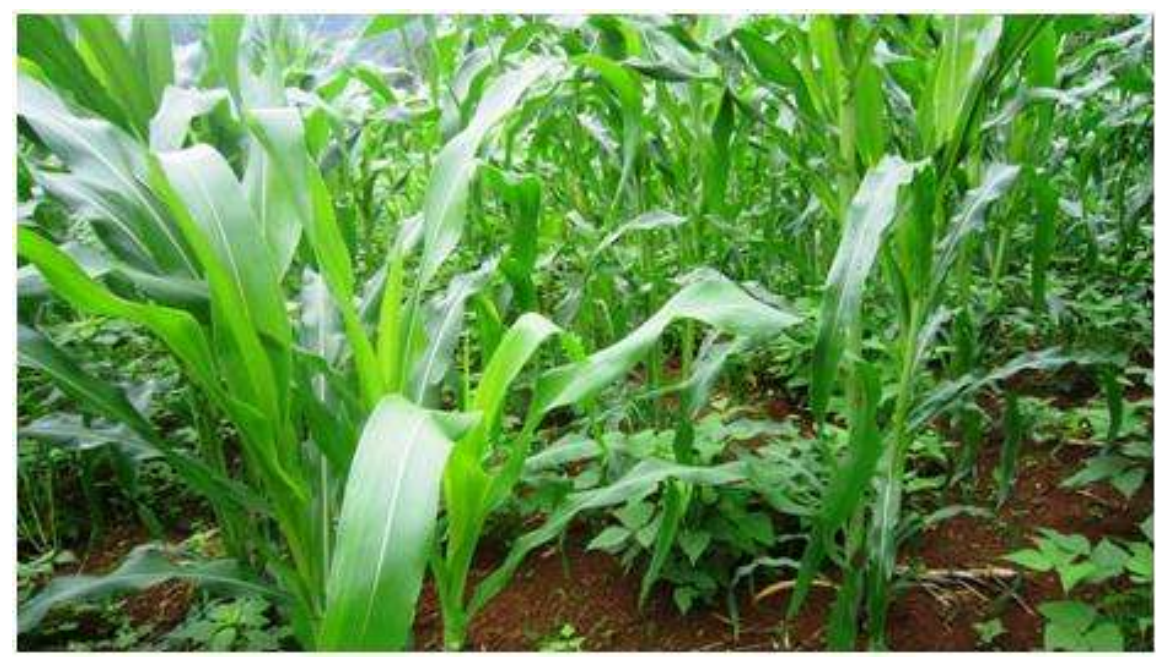

Figure 7. Intercropped maize and beans by H'Mong people in Ta Phin, Tua Chua, Dien Bien 
In addition to those mentioned, the main challenges at this elevation were poor infrastructure, low education levels and limited access to services and information owing to the relatively remote locations.

\section{Input levels}

The input levels differed greatly among the 17 villages, depending on household budgets. For example, in lowland areas where maize was cultivated for commercial purposes, farmers applied 1$1.3 \mathrm{t}$ /ha inorganic fertilizer (NPK) for a production level of 6-7 t/ha. By contrast, in Quai Nua (Thai) commune and in H'mong villages, the input levels were significantly lower: 0-0.2 t/ha, with results of 1.5-2 $\mathrm{t} / \mathrm{ha}$. The low productivity at higher elevations was also due to climatic conditions and poor access to markets.

\section{Economic effectiveness of some dominant farming systems in the study villages}

The profits obtained from the farming practices of upland farmers were relatively low and oftentimes the total income was only just enough to cover the inputs (material and labour). There were several explanations for this: 1) the estimated labour inputs for land preparation, planting, weeding and harvesting for farms that have fragmented fields - some are sloping, distant and difficult to accesscan be greater compared to those for farms with more easily accessible fields; 2) in response to declining soil fertility, farmers increased doses of fertilizer but improvements in yields could not compensate for increased production costs; 3) yields of local varieties were generally lower than improved varieties, in addition, the quality of local upland rice and maize varieties seemed to have degenerated, further resulting in declining yields; 4) farmers primarily sold unprocessed, raw products at unstable prices; in many cases, limited market access and storage capacity drove farmers to sell at low prices. The estimated economic investments, turnover and profitability of different farming systems are shown in table 5 .

Overall, the most profitable systems at high elevations were shan tea with cassava in Suoi Giang, Van Chan (VND 20 million ( \pm USD 955) per year) followed by Coix Lacryma-jobi in Toa Tinh, Tuan Giao (VND 10 million ( \pm USD 480) ha/year in 2011) and maize intercropped with pumpkins in Pang Cang (VND 7.6 million ( \pm USD 363)). At intermediate elevations, coffee in Chieng Bom (VND 15 million $( \pm$ USD 717)), upland rice (VND 13.5 million ( \pm USD 645$)$ ) and maize (VND 12.5 million $( \pm$ USD 597)) in Co Noi, Mai Son, were the most profitable. At low elevations, tomato (VND 27 million ( \pm USD 1290)) and sugar cane (VND 9 million ( \pm USD 430)). The least profitable systems were upland rice in Co Ma, Thuan Chau, and in Suoi Giang, Van Chan, and the paddy rice systems in general (VND 0.55 million ( \pm USD 26$)$ ). 
Table 5. Economic effectiveness of selected farming systems

\begin{tabular}{|c|c|c|c|c|c|c|c|}
\hline District & Commune & Village & $\begin{array}{l}\text { Farming } \\
\text { system }\end{array}$ & $\begin{array}{l}\text { Investment/all } \\
\text { inputs } \\
\text { (VND/ha) }\end{array}$ & $\begin{array}{l}\text { Total } \\
\text { income/turnover } \\
\text { (VND/ha) }\end{array}$ & $\begin{array}{l}\text { Profit of } \\
\text { individual } \\
\text { components } \\
\text { (VND/ha) }\end{array}$ & $\begin{array}{l}\text { System profit } \\
\text { (VND/ha/year) }\end{array}$ \\
\hline \multicolumn{8}{|l|}{$<600 \mathrm{~m}$} \\
\hline \multirow{6}{*}{$\begin{array}{l}\text { Van } \\
\text { Chan }\end{array}$} & \multirow[t]{6}{*}{ Son Thinh } & \multirow[t]{2}{*}{ Van Thi 4} & Maize monocrop & $10,955,000$ & $12,000,000$ & & $1,045,000$ \\
\hline & & & $\begin{array}{l}\text { Cassava } \\
\text { monocrop }\end{array}$ & $14,450,000$ & $17,500,000$ & & $3,050,000$ \\
\hline & & \multirow[t]{4}{*}{ Hong Son } & Maize & $21,700,000$ & $27,500,000$ & $5,800,000$ & $5,800,000$ \\
\hline & & & Sugar cane & $26,050,000$ & $35,000,000$ & $8,950,000$ & $8,950,000$ \\
\hline & & & Tomato & $23,700,000$ & $50,400,000$ & $26,700,000$ & $26,700,000$ \\
\hline & & & Peanuts & $11,550,000$ & $18,000,000$ & $6,450,000$ & $6,450,000$ \\
\hline \multicolumn{8}{|c|}{$600-800 \mathrm{~m}$} \\
\hline \multirow[t]{9}{*}{ Mai Son } & \multirow[t]{8}{*}{ Co Noi } & \multirow[t]{2}{*}{ Phieng Hy } & Maize & $20,155,000$ & $24,000,000$ & & $7,845,000$ \\
\hline & & & Upland rice & $16,520,000$ & $33,000,000$ & & $13,480,000$ \\
\hline & & \multirow[t]{6}{*}{ Mon } & Maize monocrop & $22,300,000$ & $34,800,000$ & $12,500,000$ & $12,500,000$ \\
\hline & & & Rice & $26,220,000$ & $31,500,000$ & $5,280,000$ & $5,280,000$ \\
\hline & & & Sugar cane & $37,620,000$ & $47,500,000$ & $9,880,000$ & $9,880,000$ \\
\hline & & & Mango & $12,580,000$ & $14,000,000$ & $1,420,000$ & $1,420,000$ \\
\hline & & & Coffee ( $3^{\text {rd }}$ year $)$ & $18,120,000$ & $25,000,000$ & $6,880,000$ & $6,880,000$ \\
\hline & & & Cassava & $10,450,000$ & $14,400,000$ & $3,950,000$ & $3,950,000$ \\
\hline & $\begin{array}{l}\text { Chieng } \\
\text { Chan }\end{array}$ & Sai Luong & Maize monocrop & $19,400,000$ & $29,250,000$ & $9,850,000$ & $9,850,000$ \\
\hline \multirow{2}{*}{$\begin{array}{l}\text { Thuan } \\
\text { Chau }\end{array}$} & \multirow{2}{*}{$\begin{array}{l}\text { Chieng } \\
\text { Bom }\end{array}$} & \multirow[t]{2}{*}{ Nhop } & Coffee $\left(4^{\text {th }}\right.$ year $)$ & $40,200,000$ & $55,000,000$ & & $14,800,000$ \\
\hline & & & Cassava & $5,600,000$ & $8,400,000$ & & $2,800,000$ \\
\hline \multirow{7}{*}{$\begin{array}{l}\text { Tuan } \\
\text { Giao }\end{array}$} & \multirow[t]{7}{*}{ Quai Nua } & \multirow[t]{4}{*}{ Cha } & Maize & $14,900,000$ & $12,500,000$ & $-2,400,000$ & \multirow[t]{2}{*}{$5,900,000$} \\
\hline & & & Peanut & $14,100,000$ & $22,400,000$ & $8,300,00$ & \\
\hline & & & Paddy & $8,600,000$ & 9000.000 & 400.000 & 8.700 .000 \\
\hline & & & Peanut & 14.100 .000 & 22.400 .000 & 8.300 .000 & \\
\hline & & \multirow[t]{3}{*}{ Ban Chan } & Maize & $14,255,000$ & $21,000,000$ & $6,745,000$ & $6,745,000$ \\
\hline & & & Paddy & $26,870,000$ & $28,000,000$ & $1,130,000$ & $1,130,000$ \\
\hline & & & Cassava & $15,710,000$ & $21,250,000$ & $5,540,000$ & $5,540,000$ \\
\hline \multicolumn{8}{|l|}{$>800 \mathrm{~m}$} \\
\hline \multirow{6}{*}{$\begin{array}{l}\text { Thuan } \\
\text { Chau }\end{array}$} & \multirow[t]{2}{*}{ Long $\mathrm{He}$} & \multirow{2}{*}{$\begin{array}{l}\text { Nong Coc } \\
\text { A }\end{array}$} & Upland rice & $8,250,000$ & $10,400,000$ & $2,150,000$ & $2,150,000$ \\
\hline & & & Cassava & $20,910,000$ & $24,000,000$ & $3,090,000$ & $3,090,000$ \\
\hline & \multirow[t]{4}{*}{ Co Ma } & \multirow[t]{4}{*}{ Co Ma } & \multirow{2}{*}{$\begin{array}{l}\text { Maize \& } \\
\text { Pumpkin }\end{array}$} & $10,305,000$ & $14,400,000$ & $4,095,000$ & $5,120,000$ \\
\hline & & & & $1,375,000$ & $2,400,000$ & $1,025,000$ & \\
\hline & & & Upland rice \& & $8,250,000$ & $8,800,000$ & 550,000 & 975,000 \\
\hline & & & $\begin{array}{l}\text { H'mong } \\
\text { cucumber }\end{array}$ & 575,000 & $1,000,000$ & 425,000 & \\
\hline & & & $\begin{array}{l}\text { Pine }\left(7^{\text {th }} \text { year, resin }\right. \\
\text { harvesting })\end{array}$ & $7,232,000$ & $6,000,000$ & $-1,232,000$ & $-508,000$ \\
\hline Tuan & Toa Tinh & Hua Sa A & Son tra $\left(7^{\text {th }}\right.$ year $)$ & $6,332,000$ & $7,056,000$ & 724,000 & \\
\hline Giao & & & $\begin{array}{l}\text { Coix lacryma- } \\
\text { jobi }\end{array}$ & 5,980 & $16,008,000$ & & $10,028,000$ \\
\hline & & & $\begin{array}{l}\text { Purple Amomum } \\
\left(3^{\text {rd }} \text { year }\right)\end{array}$ & $24,000,000$ & $27,000,000$ & & $3,000,000$ \\
\hline & & Che A & Upland rice & $7,360,000$ & $9,600,000$ & $2,240,000$ & $2,240,000$ \\
\hline & & & Maize & $9,305,000$ & $15,600,000$ & $6,295,000$ & $6,295,000$ \\
\hline
\end{tabular}




\begin{tabular}{|c|c|c|c|c|c|c|c|}
\hline & & & Cassava & $16,380,000$ & $22,500,000$ & $6,120,000$ & $6,120,000$ \\
\hline \multirow{3}{*}{$\begin{array}{l}\text { Tua } \\
\text { Chua }\end{array}$} & \multirow[t]{3}{*}{ Ta phin } & \multirow[t]{3}{*}{ Ta Phin 2} & Maize & $11,655,000$ & $12,600,000$ & & 945,000 \\
\hline & & & Upland rice & $20,265,000$ & $24,000,000$ & & $3,735,000$ \\
\hline & & & $\begin{array}{l}\text { Cardamom }\left(3^{\text {rd }}\right. \\
\text { year })\end{array}$ & 9.600 .000 & 2.400 .000 & & -7.200 .000 \\
\hline \multirow{7}{*}{$\begin{array}{l}\text { Van } \\
\text { Chan }\end{array}$} & \multirow[t]{7}{*}{ Suoi Giang } & \multirow[t]{3}{*}{ Giang B } & Maize & 10.680 .000 & 12.000 .000 & & 1.320 .000 \\
\hline & & & Shan tea & 4.500 .000 & 4.200 .000 & & -300.000 \\
\hline & & & ( $3^{\text {rd }}$ year) & & & & \\
\hline & & \multirow{4}{*}{$\begin{array}{l}\text { Pang } \\
\text { Cang }\end{array}$} & \multirow{2}{*}{$\begin{array}{l}\text { Maize \& } \\
\text { pumpkin }\end{array}$} & $9,655,000$ & $16,200,000$ & $6,545,000$ & \multirow[t]{2}{*}{$7,595,000$} \\
\hline & & & & $1,650,000$ & $2,700,000$ & $1,050,000$ & \\
\hline & & & \multirow{2}{*}{$\begin{array}{l}\text { Shan tea } \\
\text { \& cassava }\end{array}$} & $13,000,000$ & $32,000,000$ & $19,000,000$ & \multirow[t]{2}{*}{$20,250,000$} \\
\hline & & & & $7,750,000$ & $9,000,000$ & $1,250,000$ & \\
\hline $\begin{array}{l}\text { Trạm } \\
\text { Tau }\end{array}$ & Cong & Ta Xua & $\begin{array}{l}\text { Maize \& } \\
\text { pumpkin }\end{array}$ & $10,225,000$ & $13,100,000$ & & $2,875,000$ \\
\hline
\end{tabular}

The profit from maize cultivation varied from VND 945,000 ha/year above 800 masl to VND 12,500,000/ha at the intermediate elevation where the climatic conditions (temperature and rainfall) seem appropriate for hybrid maize varieties.

Although paddy and upland rice made minor contributions to household incomes (VND 500,000 ha/year) the harvest is indispensable for ensuring household food security.

Cassava mono-cultivation also generated comparatively low incomes, from VND 3,090,000 to VND 6,020,000 ha/year. However, cassava provided an alternative to rice and maize as soil fertility began to decline.

Coffee and shan tea showed relatively high economic returns in some areas, up to VND 20,000,000 ha/year. In addition, coffee and shan tea were among the species that farmers preferred to gradually replace maize when soils had degraded. Coffee and shan tea generated returns from the third year of planting, if planted as seedlings.

Cardamom planted under forest canopy did not show profit until the third year.

\section{Potential plant species by agro-ecological zone}

Species that the interviewed farmers considered had potential for being integrated into an agroforestry system are shown in table 6.

Below 600 masl, farmers preferred eucalyptus and acacia because of their suitability to the natural conditions and local demand for timber. Of the fruit tree species, farmers preferred late-fruiting longan and orange for marketability. 
Table 6. Species with high potential for agroforestry by agro-ecological zone

\begin{tabular}{|c|c|c|}
\hline Elevation & Species & Reason \\
\hline \multirow[t]{3}{*}{$<600 \mathrm{~m}$} & $\begin{array}{l}\text { Hybrid eucalyptus, } \\
\text { Acacia } \\
\text { auriculiformis }\end{array}$ & $\begin{array}{l}\text { In Son La and Dien Bien, these species are in demand for house } \\
\text { construction because natural wood resources have been overexploited }\end{array}$ \\
\hline & Late-fruiting longan & This variety gives high fruit quality and late harvesting season \\
\hline & Orange & Traditionally grown in Yen Bai with relative good market price \\
\hline \multirow[t]{4}{*}{$600-800 \mathrm{~m}$} & Coffee & $\begin{array}{l}\text { Can give high economic returns but requires high investment for } \\
\text { establishment and annual inputs. Many farmers have been successful } \\
\text { with coffee plantations }\end{array}$ \\
\hline & Macadamia & High potential for income generation if able to enter international market \\
\hline & Canarium nigrum & Multipurpose native species gives high value nuts and timber \\
\hline & Eucalyptus & In demand for local use (house construction) \\
\hline \multirow[t]{6}{*}{$>800 \mathrm{~m}$} & Son tra & $\begin{array}{l}\text { Native tree species, provides fruit for cash and home consumption, } \\
\text { shade, soil protection and timber. It can be intercropped with timber } \\
\text { species or crops. Good market opportunities }\end{array}$ \\
\hline & Shan tea & $\begin{array}{l}\text { Considered a good investment owing to long life (100+ years), high yield } \\
\text { and good quality of tea (well-developed market locally and for export in } \\
\text { Yen Bai) }\end{array}$ \\
\hline & French peach & Can be grown at high elevations on sloping land \\
\hline & Walnut & $\begin{array}{l}\text { Grows well at high elevations (Co Ma), gives high yield, good market } \\
\text { opportunities }\end{array}$ \\
\hline & $\begin{array}{l}\text { Cunminghamia } \\
\text { lanceolata Lamb } \\
\text { ('sa moc') }\end{array}$ & Provides timber for house construction, can grow well at high elevation \\
\hline & $\begin{array}{l}\text { Amomum } \\
\text { Cardamom }\end{array}$ & Planted under forest canopy, good market opportunities \\
\hline
\end{tabular}

At 600-800 masl, farmers support eucalyptus and Canarium. Canarium is a multipurpose species with long rotation that provides nuts and, eventually, high quality timber. Some plant Canarium as a shade tree for coffee. Coffee was preferred in Son La and Dien Bien provinces since it brings higher economic returns than traditional maize and cassava cultivation. However, as coffee has a high initial investment only better-off farmers can afford the system without loans.

Macadamia was a newly introduced species that grows well in the Northwest with good yields ( $4 \mathrm{~kg} /$ tree in the $8^{\text {th }}$ year) (Mai Son district) and potential for export to international markets.

At high elevations, farmers prefer temperate timber and fruit/nut species, including son tra, French peach or shan tea. Walnut has shown good growth in Thuan Chau above 1100 masl, and could be one option. Amomum with cardamom can be considered as a component under tree canopies.

\section{Profile of farmer co-operators}

\section{Household characteristics}

Of the total of 45 interviewed farmer co-operators, 42 were men and three were women. Agriculture was the main income source for over $95 \%$ of the farmer co-operators and the main cash crops were maize $(82 \%)$, paddy rice, coffee or tea (18\%) (figure 8 ). 
More than half of the interviewees were in middle income households, $12 \%$ had high incomes, and the remaining (36\%) were in poor households (as classified in the village household list (figure 9). The average households' size was 5 and average number of people in working age per household was 3.

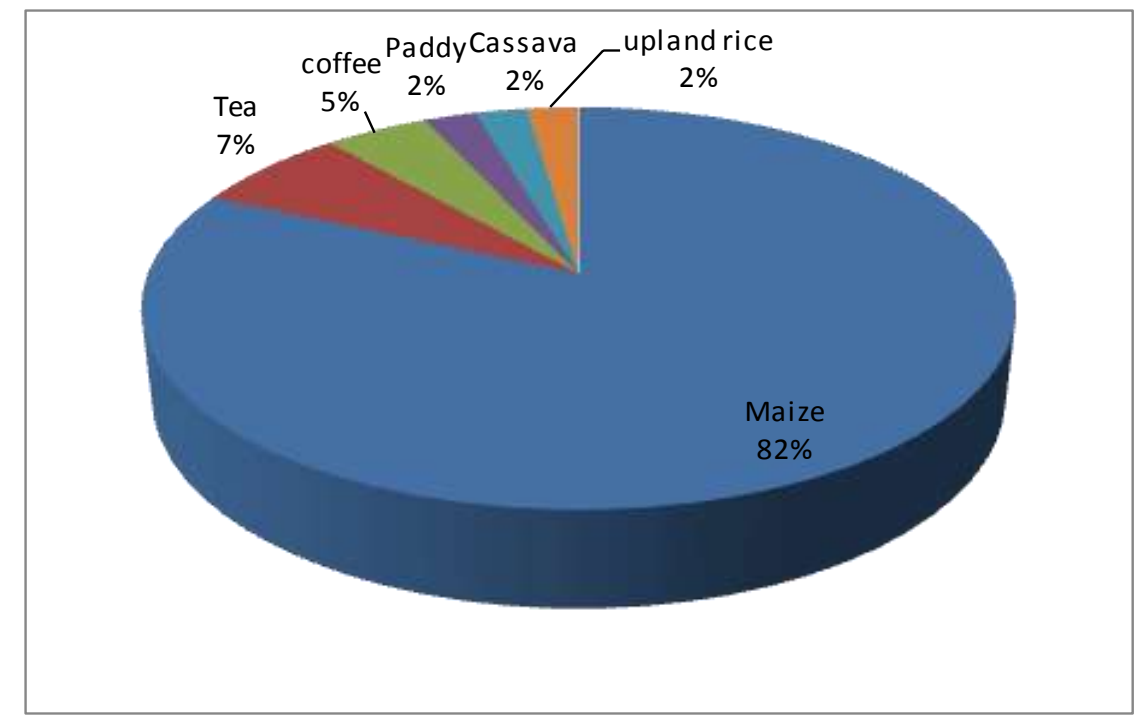

Figure 8. Crops provide the main income for households

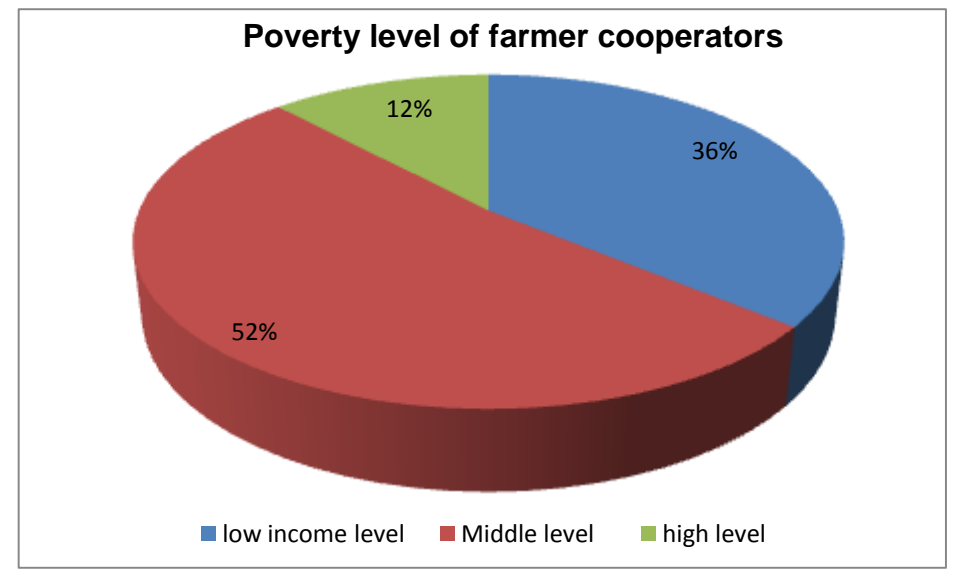

Figure 9. Income levels of farmer co-operators $(n=45)$

\section{Land and agricultural farming practices}

Nearly $20 \%$ of the interviewees owned 5-19 ha of agricultural land, $42 \%$ had $2-4$ ha, $31 \%$ had less than 2 ha and 7\% did not possess any land and farmed on rented land. Land boundaries were demarcated in most fields with a row of trees or bushes, grass strips or stone fences.

The distance between houses and fields was less than $3 \mathrm{~km}(70 \%)$ and 3-5 km (30\%). The fields were accessed by motorbike, if available. 
Chemical plant protection substrates (mainly pesticide) were used by $75 \%$ of the farmer co-operators. In general, they used fewer chemicals for maize cultivation than for upland and paddy rice, applied herbicide once at the beginning of the planting season, if any, and only rarely during the growing period. For rice cultivation, farmers often used chemicals against Scirpophaga incertulas Walker (Yellow stem borer). Most could not remember the name of the chemicals. In Ban Cong commune, Tram Tau district and Son Thinh commune, Van Chan district, the farmer co-operators used no plant protection.

Figure 10 shows that the most important factors for the farmers in deciding which trees and/or crops to plant or which farming system and structure to apply were the product market, land availability and capital resources.

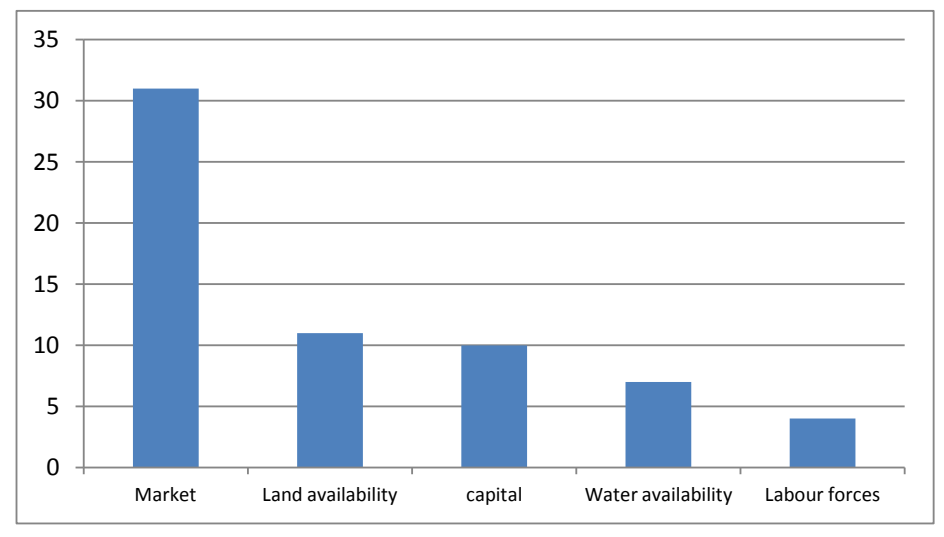

Figure 10. Most important factors in farmers' decisions on what trees and/or crops to plant $(\mathrm{n}=45)$

The main factors for good yields, according to farmers, were, firstly, limited soil erosion (mentioned by $85 \%$ or 38 of the 45 interviewed), followed by water resources, particularly availability (65\% or 29 out of the 45 ) and soil fertility ( $52 \%$ or 23 out of the 45 ). Only three farmers mentioned inputs and three mentioned health factors. It is clear that soil erosion and declining fertility and water availability are a significant problem in the region.

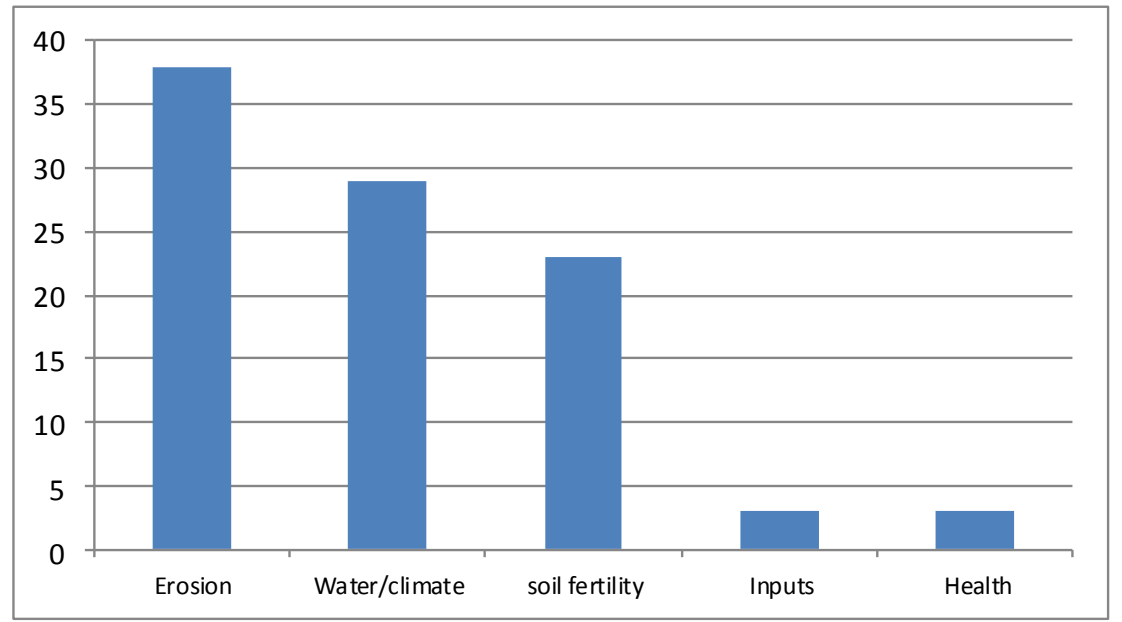

Figure 11. Factors limiting productivity, according to farmers $(n=45)$ 
Regarding the changes in the determinant factors for good yields in the last five years that were reported by farmers: the first was declining soil fertility and soil erosion followed by higher input levels.

\section{Tree-planting and agroforestry practices}

Prior to the project, $70 \%$ of the farmer co-operators already had trees on their farms for home consumption and additional income. The most common fruit tree species were mango, litchi, longan, plum, peach, son tra. The most common timber species were melia, acacia, pine, Vernicia Montana, Manglietia conifer, Fokienia hodginsii and bamboo species.

Most trees were planted in home gardens or scattered around the farm: at the top of a hill, on foothills or along the contours of slopes.

The challenges with managing trees were limited land resources, limited selection of species with high economic potential (market demand), lack of investment capital, pests and diseases. Other problems mentioned by the interviewed farmers were water availability, grazing and insufficient labor resources.

The farmer co-operators had limited knowledge about agroforestry. Prior to the project, none had participated in an agroforestry project. Few had even heard the term 'agroforestry', however, after providing some examples 36 of 44 farmer co-operators (82\%) understood agroforestry technologies, such as intercropping, taungya, garden-fishpond-cage or forest-garden-fishpond-cage, and 15 farmers said they practised some kind of agroforestry on their farms.

A training needs assessment of extension workers showed that one reason for the limited uptake of agroforestry systems was that the extension network was not as active as the farmers wanted and the commune workers were not trained in agroforestry. Rather, they typically specialize and/or have experience in either agriculture or forestry and only with a few species.

The main sources from which the farmers learnt about agroforestry were family members and neighbours (45\%) and mass media (TV, radio, newspapers) (28\%).

All the farmer co-operators were willing to plant more trees on their farm in order to reverse low soil fertility, increase soil protection and benefit from the long-term stability of tree-based systems. However, they were concerned about the availability of species of high economic benefit and the availability of markets.

\section{Forestry and livestock}

About one-third of the farmer co-operators had been allocated forestland. The forestland area generally varied 0.1-3 ha per household, with the exception of one household with a 15 ha plantation and 12 ha of natural forest. Only a few households received additional income from their forestland by intercropping with food crops or NTFPs, from contracting for forest protection, through payments for environmental services' scheme or selling firewood.

Nearly $90 \%$ of the farmers raised animals, in particular, buffalo, cows, goats and poultry. Of the 34 farmers raising cattle, over two-thirds practised free grazing on natural grasslands while the remaining third used a cut-and-carry system to feed on-farm with natural or planted fodder grasses, particularly 
elephant grass and VA-06. The most frequent problems concerning animal husbandry were diseases $(64 \%)$, shortage of fodder grass (54\%), shortage of labour (23\%) and weather conditions (15\%).

\section{Farmers' knowledge of market information}

All the farmer co-operators sold some of their produce at home (80\%), at the farm gate (35\%), local markets $(32 \%)$ and at traders' buying stations (10\%). Most had studied market prices before selling to collectors or traders.

The difficulties that farmers encountered when selling their agricultural products were 1) unstable prices; and 2) being forced to sell at low prices owing to shortages of cash or lack of storage. Farmers encountered difficulties in finding markets for their produce and transporting it owing to poor road conditions.

\section{Gender and labour allocation}

About 95\% of farmer co-operators said that within their households final decisions relating to farming practices, such as what and when to plant and what to sell at what prices, were primarily made by the men after consultation with their wives. In terms of labour division, $80 \%$ stated that soil preparation, planting and tending was done by all family members. Feeding and looking after animals was done by children, women and elderly people.

\section{Discussion and conclusion}

The dominant farming system in all three agro-ecological zones in the three northwestern provinces was mono-cultivation of staple crops on sloping land. The poor diversity of farming system structure and components made smallholders vulnerable to market fluctuation. Furthermore, the risk of pests and crop failure in a monocropping system was high (Lithourgidis 2011). More diversified farming systems and crop patterns could be one of the sustainable solutions that provides farmers with more income options and contributes to food security during winter (Davis 2012). Agroforestry is one of these solutions: it can diversify farming products while addressing environmental problems.

Furthermore, soil degradation as a result of mono-cultivation, high soil erosion and intensive farming practices, along with water scarcity, made it difficult for farmers to maintain crop productivity. Farmers were willing to change their farming practices if a better alternative was available. Some of them started planting Manglietia, intercropped with food crops in the first years when the tree canopy was still open.

Development of agroforestry in the region is likely to be a good option for restoring soil fertility, improving the environmental functions of the ecosystem and enhancing the performance of farming systems (Young 1989). Studies also show that simple measures, such as grass strips along contours (Phien et al 2012) or green cover with multipurpose species like Arachis pintoi (Argel et al 2005) could be used as green mulch to improve soil fertility and reduce erosion for conservation purposes. Grass can also be a good fodder resource for cattle, which in turn can provide additional income. Given the low income levels of local farmers, agroforestry systems that combine staple crops with short-term returns and trees giving middle- and long-term income have high potential for adoption. To help curb water scarcity, small farm reservoirs, water impounding structures and other rainwater harvesting techniques could be deployed (Bhuiyan et al 1994). 
With regard to agroforestry, sustainable tree-based farming systems on sloping land are not yet developed in the study areas. Traditional agroforestry systems have existed in some locations but at very small scale. Farmers and extension workers' knowledge about sustainable farming and agroforestry practices was limited. The limited adoption of agroforestry in the study areas could be partly due to limited activities, or capacity in, agroforestry of the local extension network. In order to deliver the message about sustainable agroforestry to the region, the extension networks at the local level should be strengthened. It is to recommend that along with the implementation of on-farm research trials, a capacity-building program in agroforestry for the extension network should be carried out.

Farmers in the study village were willing to plant more trees but were concerned about species that have high market potential and the amount of land needed. Agroforestry can only be promoted if important preconditions for wider adoption are ensured (Wood 2012); the most essential being availability of markets and land. We also recommend that, along with promoting the adoption and expansion of agroforestry, support is provided to develop markets and processing techniques for agroforestry products.

The list of farmers' preferred species (table 6) included species that were selected for the trials in the project: late-fruiting longan, macadamia, son tra, shan tea and Amomum. This confirmed that the selected species were relevant to local conditions. However, given the severity of soil degradation and erosion in the study areas and the tree densities designed for the trials ( 4 x $4 \mathrm{~m}$ or $5 \times 5 \mathrm{~m})$, the project should consider additional measures to reduce erosion and restore soil fertility while the trees are still small. Grass strips and green groundcover could be suitable solutions.

To meet the local demand for fast-growing timber for construction, eucalyptus trees or others could be scatter-planted with crops.

Home gardens at high elevations could be improved by adding temperate fruit and nut tree species with economic potential, such as persimmon and French peach or walnut.

In summary, agroforestry has great potential to be promoted in the study areas and it is also a suitable time for any intervention or farming innovation. However, given the low investment capacity of farmers, the fragmentation of land resources and the limited knowledge of extension workers and farmers about agroforestry, the adoption of agroforestry will be challenging. Incentives policy in agroforestry and land tenure should be designed and implemented along with other technical measures. 


\section{References}

Argel PJ, Kerridge PJ, Pizarro EA. 2005. Arachis pintoi: A multipurpose legume for sustainable land use. Cali, Columbia: Tropical Forage Program, Centro International de Agricultura Tropical.

Bhuiyan SI, ed. 1994. On-farm reservoir systems for rainfed ricelands. Manila: International Rice Research Institute.

Davis AS, Hill JD, Chase CA, Johanns AM, Liebman M. 2012. Increasing cropping system diversity balances productivity, profitability and environmental health. PLOS ONE 7(10):e47149.

Hoang MH, Johansen MD, Degrande A, Luu TG, Roshetko J, Mulyoutami E. 2010. Son La scoping study report. Hanoi, Viet Nam: World Agroforestry Centre (ICRAF) Viet Nam.

Lithourgidis AS, Dordas CA, Damalas CA, Vlachostergios DN. 2011. Annual intercrops: an alternative pathway for sustainable agriculture. Australian Journal of Crop Science 5(4):396-410

Phien T, Tran TT. Vetiver grass in hedgerow farming systems on sloping lands in Vietnam. Hanoi, Viet Nam: National Institute for Soils and Fertilizers. Available from http://www.vetiver.org/VNN_Thai\%20Phien.pdf.

Woods P, Petheram RJ. 2001. Pre-conditions for Spontaneous Agroforestry in Hilly Regions of Viet Nam: Implications for extension. Melbourne, Australia: Institute of Land and Food Resources, University of Melbourne.

Young A. 1989. Agroforestry for soil conservation. Wallingford, UK: CABI; Nairobi: International Council for Research in Agroforestry. 


\section{WORKING PAPERS IN THIS SERIES}

2005

1. Agroforestry in the drylands of eastern Africa: a call to action

2. Biodiversity conservation through agroforestry: managing tree species diversity within a network of community-based, nongovernmental, governmental and research organizations in western Kenya.

3. Invasion of prosopis juliflora and local livelihoods: Case study from the Lake Baringo area of Kenya

4. Leadership for change in farmers organizations: Training report: Ridar Hotel, Kampala, 29th March to 2nd April 2005.

5. Domestication des espèces agroforestières au Sahel : situation actuelle et perspectives

6. Relevé des données de biodiversité ligneuse: Manuel du projet biodiversité des parcs agroforestiers au Sahel

7. Improved land management in the Lake Victoria Basin: TransVic Project's draft report.

8. Livelihood capital, strategies and outcomes in the Taita hills of Kenya

9. Les espèces ligneuses et leurs usages: Les préférences des paysans dans le Cercle de Ségou, au Mali

10. La biodiversité des espèces ligneuses: Diversité arborée et unités de gestion du terroir dans le Cercle de Ségou, au Mali

2006

11. Bird diversity and land use on the slopes of Mt. Kilimanjaro and the adjacent plains, Tanzania

12. Water, women and local social organization in the Western Kenya Highlands

13. Highlights of ongoing research of the World Agroforestry Centre in Indonesia

14. Prospects of adoption of tree-based systems in a rural landscape and its likely impacts on carbon stocks and farmers' welfare: The FALLOW Model Application in Muara Sungkai, Lampung, Sumatra, in a 'Clean Development Mechanism' context

15. Equipping integrated natural resource managers for healthy Agroforestry landscapes.

17. Agro-biodiversity and CGIAR tree and forest science: approaches and examples from Sumatra.

18. Improving land management in eastern and southern Africa: A review of policies.

19. Farm and household economic study of Kecamatan Nanggung, Kabupaten Bogor, Indonesia: A socio-economic base line study of Agroforestry innovations and livelihood enhancement.

20. Lessons from eastern Africa's unsustainable charcoal business.

21. Evolution of RELMA's approaches to land management: Lessons from two decades of research and development in eastern and southern Africa

22. Participatory watershed management: Lessons from RELMA's work with farmers in eastern Africa.

23. Strengthening farmers' organizations: The experience of RELMA and ULAMP.

24. Promoting rainwater harvesting in eastern and southern Africa.

25. The role of livestock in integrated land management.

26. Status of carbon sequestration projects in Africa: Potential benefits and challenges to scaling up. 
27. Social and Environmental Trade-Offs in Tree Species Selection: A Methodology for Identifying Niche Incompatibilities in Agroforestry [Appears as AHI Working Paper no. 9]

28. Managing tradeoffs in agroforestry: From conflict to collaboration in natural resource management. [Appears as AHI Working Paper no. 10]

29. Essai d'analyse de la prise en compte des systemes agroforestiers pa les legislations forestieres au Sahel: Cas du Burkina Faso, du Mali, du Niger et du Senegal.

30. Etat de la recherche agroforestière au Rwanda etude bibliographique, période 19872003

31. Science and technological innovations for improving soil fertility and management in Africa: A report for NEPAD's Science and Technology Forum.

32. Compensation and rewards for environmental services.

33. Latin American regional workshop report compensation.

34. Asia regional workshop on compensation ecosystem services.

35. Report of African regional workshop on compensation ecosystem services.

36. Exploring the inter-linkages among and between compensation and rewards for ecosystem services CRES and human well-being

37. Criteria and indicators for environmental service compensation and reward mechanisms: realistic, voluntary, conditional and pro-poor

38. The conditions for effective mechanisms of compensation and rewards for environmental services.

39. Organization and governance for fostering Pro-Poor Compensation for Environmental Services.

40. How important are different types of compensation and reward mechanisms shaping poverty and ecosystem services across Africa, Asia \& Latin America over the Next two decades?

41. Risk mitigation in contract farming: The case of poultry, cotton, woodfuel and cereals in East Africa.

42. The RELMA savings and credit experiences: Sowing the seed of sustainability

43. Yatich J., Policy and institutional context for NRM in Kenya: Challenges and opportunities for Landcare.

44. Nina-Nina Adoung Nasional di So! Field test of rapid land tenure assessment (RATA) in the Batang Toru Watershed, North Sumatera.

45. Is Hutan Tanaman Rakyat a new paradigm in community based tree planting in Indonesia?

46. Socio-Economic aspects of brackish water aquaculture (Tambak) production in Nanggroe Aceh Darrusalam.

47. Farmer livelihoods in the humid forest and moist savannah zones of Cameroon.

48. Domestication, genre et vulnérabilité : Participation des femmes, des Jeunes et des catégories les plus pauvres à la domestication des arbres agroforestiers au Cameroun.

49. Land tenure and management in the districts around Mt Elgon: An assessment presented to the Mt Elgon ecosystem conservation programme.

50. The production and marketing of leaf meal from fodder shrubs in Tanga, Tanzania: A pro-poor enterprise for improving livestock productivity.

51. Buyers Perspective on Environmental Services (ES) and Commoditization as an approach to liberate ES markets in the Philippines.

52. Towards Towards community-driven conservation in southwest China: Reconciling state and local perceptions. 
53. Biofuels in China: An Analysis of the Opportunities and Challenges of Jatropha curcas in Southwest China.

54. Jatropha curcas biodiesel production in Kenya: Economics and potential value chain development for smallholder farmers

55. Livelihoods and Forest Resources in Aceh and Nias for a Sustainable Forest Resource Management and Economic Progress

56. Agroforestry on the interface of Orangutan Conservation and Sustainable Livelihoods in Batang Toru, North Sumatra.

57. Assessing Hydrological Situation of Kapuas Hulu Basin, Kapuas Hulu Regency, West Kalimantan.

58. Assessing the Hydrological Situation of Talau Watershed, Belu Regency, East Nusa Tenggara.

59. Kajian Kondisi Hidrologis DAS Talau, Kabupaten Belu, Nusa Tenggara Timur.

60. Kajian Kondisi Hidrologis DAS Kapuas Hulu, Kabupaten Kapuas Hulu, Kalimantan Barat.

61. Lessons learned from community capacity building activities to support agroforest as sustainable economic alternatives in Batang Toru orang utan habitat conservation program (Martini, Endri et al.)

62. Mainstreaming Climate Change in the Philippines.

63. A Conjoint Analysis of Farmer Preferences for Community Forestry Contracts in the Sumber Jaya Watershed, Indonesia.

64. The highlands: a shared water tower in a changing climate and changing Asia

65. Eco-Certification: Can It Deliver Conservation and Development in the Tropics.

66. Designing ecological and biodiversity sampling strategies. Towards mainstreaming climate change in grassland management.

67. Towards mainstreaming climate change in grassland management policies and practices on the Tibetan Plateau

68. An Assessment of the Potential for Carbon Finance in Rangelands

69 ECA Trade-offs Among Ecosystem Services in the Lake Victoria Basin.

69. The last remnants of mega biodiversity in West Java and Banten: an in-depth exploration of RaTA (Rapid Land Tenure Assessment) in Mount Halimun-Salak National Park Indonesia

70. Le business plan d'une petite entreprise rurale de production et de commercialisation des plants des arbres locaux. Cas de quatre pépinières rurales au Cameroun.

71. Les unités de transformation des produits forestiers non ligneux alimentaires au Cameroun. Diagnostic technique et stratégie de développement Honoré Tabuna et Ingratia Kayitavu.

72. Les exportateurs camerounais de safou (Dacryodes edulis) sur le marché sous régional et international. Profil, fonctionnement et stratégies de développement.

73. Impact of the Southeast Asian Network for Agroforestry Education (SEANAFE) on agroforestry education capacity.

74. Setting landscape conservation targets and promoting them through compatible land use in the Philippines.

75. Review of methods for researching multistrata systems.

76. Study on economic viability of Jatropha curcas L. plantations in Northern Tanzania assessing farmers' prospects via cost-benefit analysis

77. Cooperation in Agroforestry between Ministry of Forestry of Indonesia and International Center for Research in Agroforestry

78. "China's bioenergy future. an analysis through the Lens if Yunnan Province

79. Land tenure and agricultural productivity in Africa: A comparative analysis of the economics literature and recent policy strategies and reforms 
80. Boundary organizations, objects and agents: linking knowledge with action in agroforestry watersheds

81. Reducing emissions from deforestation and forest degradation (REDD) in Indonesia: options and challenges for fair and efficient payment distribution mechanisms

2009

82. Mainstreaming climate change into agricultural education: challenges and perspectives

83. Challenging conventional mindsets and disconnects in conservation: the emerging role of eco-agriculture in Kenya's landscape mosaics

84. Lesson learned RATA garut dan bengkunat: suatu upaya membedah kebijakan pelepasan kawasan hutan dan redistribusi tanah bekas kawasan hutan

85. The emergence of forest land redistribution in Indonesia

86. Commercial opportunities for fruit in Malawi

87. Status of fruit production processing and marketing in Malawi

88. Fraud in tree science

89. Trees on farm: analysis of global extent and geographical patterns of agroforestry

90. The springs of Nyando: water, social organization and livelihoods in Western Kenya

91. Building capacity toward region-wide curriculum and teaching materials development in agroforestry education in Southeast Asia

92. Overview of biomass energy technology in rural Yunnan (Chinese - English abstract)

93. A pro-growth pathway for reducing net $\mathrm{GHG}$ emissions in China

94. Analysis of local livelihoods from past to present in the central Kalimantan Ex-Mega Rice Project area

95. Constraints and options to enhancing production of high quality feeds in dairy production in Kenya, Uganda and Rwanda

2010

96. Agroforestry education in the Philippines: status report from the Southeast Asian Network for Agroforestry Education (SEANAFE)

97. Economic viability of Jatropha curcas L. plantations in Northern Tanzania- assessing farmers' prospects via cost-benefit analysis.

98. Hot spot of emission and confusion: land tenure insecurity, contested policies and competing claims in the central Kalimantan Ex-Mega Rice Project area

99. Agroforestry competences and human resources needs in the Philippines

100. CES/COS/CIS paradigms for compensation and rewards to enhance environmental Services

101. Case study approach to region-wide curriculum and teaching materials development in agroforestry education in Southeast Asia

102. Stewardship agreement to reduce emissions from deforestation and degradation (REDD): Lubuk Beringin's Hutan Desa as the first village forest in Indonesia

103. Landscape dynamics over time and space from ecological perspective

104. Komoditisasi atau koinvestasi jasa lingkungan: skema imbal jasa lingkungan program peduli sungai di DAS Way Besai, Lampung, Indonesia

105. Improving smallholders' rubber quality in Lubuk Beringin, Bungo district, Jambi province, Indonesia: an initial analysis of the financial and social benefits

106. Rapid Carbon Stock Appraisal (RACSA) in Kalahan, Nueva Vizcaya, Philippines

107. Tree domestication by ICRAF and partners in the Peruvian Amazon: lessons learned and future prospects in the domain of the Amazon Initiative eco-regional program

108. Memorias del Taller Nacional: "Iniciativas para Reducir la Deforestación en la region Andino - Amazónica", 09 de Abril del 2010. Proyecto REALU Peru 
109. Percepciones sobre la Equidad y Eficiencia en la cadena de valor de REDD en Perú - Reporte de Talleres en Ucayali, San Martín y Loreto, 2009. Proyecto REALU-Perú.

110. Reducción de emisiones de todos los Usos del Suelo. Reporte del Proyecto REALU Perú Fase 1

111. Programa Alternativas a la Tumba-y-Quema (ASB) en el Perú. Informe Resumen y Síntesis de la Fase II. 2da. versión revisada

112. Estudio de las cadenas de abastecimiento de germoplasma forestal en la amazonía Boliviana

113. Biodiesel in the Amazon

114. Estudio de mercado de semillas forestales en la amazonía Colombiana

115. Estudio de las cadenas de abastecimiento de germoplasma forestal en Ecuador

116. How can systems thinking, social capital and social network analysis help programs achieve impact at scale?

117. Energy policies, forests and local communities in the Ucayali Region, Peruvian Amazon

118. NTFPs as a Source of Livelihood Diversification for Local Communities in the Batang Toru Orangutan Conservation Program

119. Studi Biodiversitas: Apakah agroforestry mampu mengkonservasi keanekaragaman hayati di DAS Konto?

120. Estimasi Karbon Tersimpan di Lahan-lahan Pertanian di DAS Konto, Jawa Timur

121. Implementasi Kaji Cepat Hidrologi (RHA) di Hulu DAS Brantas, Jawa Timur.

122. Kaji Cepat Hidrologi di Daerah Aliran Sungai Krueng Peusangan, NAD,Sumatra

123. A Study of Rapid Hydrological Appraisal in the Krueng Peusangan Watershed, NAD, Sumatra.

2011

124. An Assessment of farm timber value chains in Mt Kenya area, Kenya

125. A Comparative financial analysis of current land use systems and implications for the adoption of improved agroforestry in the East Usambaras, Tanzania

126. Agricultural monitoring and evaluation systems

127. Challenges and opportunities for collaborative landscape governance in the East Usambara Mountains, Tanzania

128. Transforming Knowledge to Enhance Integrated Natural Resource Management Research, Development and Advocacy in the Highlands of Eastern Africa

129. Carbon-forestry projects in the Philippines: potential and challenges The Mt Kitanglad Range forest-carbon development

130. Carbon forestry projects in the Philippines: potential and challenges. The Arakan Forest Corridor forest-carbon project

131. Carbon-forestry projects in the Philippines: potential and challenges. The Laguna Lake Development Authority's forest-carbon development project

132. Carbon-forestry projects in the Philippines: potential and challenges. The Quirino forest-carbon development project in Sierra Madre Biodiversity Corridor

133. Carbon-forestry projects in the Philippines: potential and challenges. The Ikalahan ancestral domain forest-carbon development

134. The Importance of Local Traditional Institutions in the Management of Natural Resources in the Highlands of Eastern Africa

135. Socio-economic assessment of irrigation pilot projects in Rwanda

136. Performance of three rambutan varieties(Nephelium lappaceum L.) on various nursery media

137. Climate change adaptation and social protection in agroforestry systems: enhancing adaptive capacity and minimizing risk of drought in Zambia and Honduras 
138. Does value chain development contribute to rural poverty reduction? Evidence of asset building by smallholder coffee producers in Nicaragua

139. Potential for biofuel feedstock in Kenya

140. Impact of fertilizer trees on maize production and food security in six districts of Malawi.

\section{2}

141. Fortalecimiento de capacidades para la gestión del Santuario Nacional Pampa Hermosa: Construyendo las bases para un manejo adaptativo para el desarrollo local. Memorias del Proyect

142. Understanding rural institutional strengthening: A cross-level policy and institutional framework for sustainable development in Kenya

143. Climate change vulnerability of agroforestry

144. Rapid assesment of the inner Niger delta of Mali

145. Designing an incentive program to reduce on-farm deforestationin the East Usambara Mountains, Tanzania

146. Extent of adoption of conservation agriculture and agroforestry in Africa: the case of Tanzania, Kenya, Ghana, and Zambia

147. Policy incentives for scaling up conservation agriculture with trees in Africa: the case of Tanzania, Kenya, Ghana and Zambia

148. Commoditized or co-invested environmental services? Rewards for environmental services scheme: River Care program Way Besai watershed, Lampung, Indonesia.

149. Assessment of the headwaters of the Blue Nile in Ethiopia.

150. Assessment of the uThukela Watershed, Kwazaulu.

151. Assessment of the Oum Zessar Watershed of Tunisia.

152. Assessment of the Ruwenzori Mountains in Uganda.

153. History of agroforestry research and development in Viet Nam. Analysis of research opportunities and gaps.

154. REDD+ in Indonesia: a Historical Perspective

155. Agroforestry and Forestry in Sulawesi series: Livelihood strategies and land use system dynamics in South Sulawesi

156. Agroforestry and Forestry in Sulawesi series: Livelihood strategies and land use system dynamics in Southeast Sulawesi.

157. Agroforestry and Forestry in Sulawesi series: Profitability and land-use systems in South and Southeast Sulawesi.

158. Agroforestry and Forestry in Sulawesi series: Gender, livelihoods and land in South and Southeast Sulawesi

159. Agroforestry and Forestry in Sulawesi series: Agroforestry extension needs at the community level in AgFor project sites in South and Southeast Sulawesi, Indonesia.

160. Agroforestry and Forestry in Sulawesi series: Rapid market appraisal of agricultural, plantation and forestry commodities in South and Southeast Sulawesi. 

The World Agroforestry Centre is an autonomous, non-profit research organization whose vision is a rural transformation in the developing world where smallholder households strategically increase their use of trees in agricultural landscapes to improve their food security, nutrition, income, health, shelter, energy resources and environmental sustainability. The Centre generates science-base knowledge about the diverse role that trees play in agricultural landscapes, and uses its research to advance policies and practices that benefit the poor and the environment.

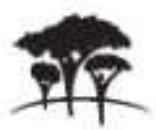

United Nations Avenue, Gigri • PO Bon 30677 - Nairobi, 00100 - Kenya Telephone: +254207224000 or via USA +16508336646 $F a x+254207224001 \propto \alpha$ vis USA +1 6508336646 Southeast Asia Regonal Program - Sindang Barang. Bogor 16115 PO Box 161 Bogor 16001, Indonesia $\mathrm{Ph}$ +62 251 B625415 - Fax: $\$ 62251$ B625416

wenw worldagroforestry org/sea 